\title{
Gyrokinetics from variational averaging: existence and error bounds
}

\author{
Stefan Possanner* \\ Technische Universität München, Zentrum Mathematik, Boltzmannstraße 3, 85748 Garching \\ Max-Planck-Institut für Plasmaphysik, Boltzmannstraße 2, 85748 Garching
}

\begin{abstract}
The gyrokinetic paradigm in the long wavelength regime is reviewed from the perspective of variational averaging (VA). The VA-method represents a third pillar for averaging kinetic equations with highly-oscillatory characteristics, besides classical averaging or Chapman-Enskog expansions. VA operates on the level of the Lagrangian function and preserves the Hamiltonian structure of the characteristics at all orders. We discuss the methodology of VA in detail by means of chargedparticle motion in a strong magnetic field. The application of VA to a broader class of highly-oscillatory problems can be envisioned. For the charged particle, we prove the existence of a coordinate map in phase space that leads to a gyrokinetic Lagrangian at any order of the expansion, for general external fields. We compute this map up to third order, independent of the electromagnetic gauge. Moreover, an error bound for the solution of the derived gyrokinetic equation with respect to the solution of the Vlasov equation is provided, allowing to estimate the quality of the VA-approximation in this particular case.
\end{abstract}

Keywords: Averaging methods, Vlasov equation, Lagrangian mechanics, motion of charged particles, magnetized plasmas.

AMS subject classifiaction: 34C29, 35Q75, 70H09, 78A35, 82D10.

\section{Introduction}

Charged particles in a strong magnetic field are spiraling around their "center of motion", the gyro-center (GY). The stronger the magnetic field, the smaller the gyro-radius $\rho_{\mathrm{s}}$ and

*stefan.possanner@ma.tum.de 
the larger the gyro-frequency $\omega_{\mathrm{c}}$ of the spiraling motion; the charged-particle dynamics is usually a multiscale problem. Low-frequency $\left(\omega / \omega_{\mathrm{c}} \ll 1\right)$ and large scale $\left(x / \rho_{\mathrm{s}} \gg 1\right)$ phenomena become of interest for instance in space physics or in magnetically confined fusion devices [30]. The modeling of these phenomena relies on averaging the gyro-motion, leading to reduced dynamics for the GY. This is a prototypical example of perturbation theory in (nearly-) periodic dynamical systems [40], with important consequences for practical applications. The GY-dynamics are appealing for numerical simulations of large ensembles of charged particles, giving rise to gyrokinetic equations. Such models are implemented in many state-of-the art computer codes for plasma turbulence simulations [7, 11, 20, 21, 27, 29, 32, 38].

Given a large ensemble of charged particles, the kinetic (Vlasov) equation for the phase space distribution $f$ of these particles reads

$$
\frac{\mathrm{d}}{\mathrm{d} t} f\left(x_{\varepsilon}(t), v_{\varepsilon}(t), t\right)=0 .
$$

The solution $f$ is constant along the characteristics $x_{\varepsilon}(t) \in \mathbb{R}^{3}, v_{\varepsilon}(t) \in \mathbb{R}^{3}$, which satisfy Newton's equations of motion under the Lorentz force. The high-dimensionality of the problem makes its numerical solution rather arduous. Moreover, in strong magnetic fields the characteristics $x_{\varepsilon}(t), v_{\varepsilon}(t)$ are highly-oscillatory with a period $\varepsilon \ll 1$, i.e. $x_{\varepsilon}(t)=x(t / \varepsilon)$ leading to severe time step restrictions in numerical solvers. Instead of following the exact trajectory, reduced dynamics for the GY have proven to be useful in numerical experiments. Gyrokinetic equations have been derived on three different levels:

1. directly from the kinetic equation (1) via a Chapman-Enskog expansion of the solution, $f=f_{0}+\varepsilon f_{1}+\ldots$,

2. from the characteristics by averaging the dynamical system $x_{\varepsilon}(t), v_{\varepsilon}(t)$,

3. on the level of the Lagrangian via "variational averaging" (VA).

In this work we shall focus on the third approach, variational averaging. VA places the emphasis on the Hamiltonian structure of the dynamical system, which is preserved in the process. The formal theory of VA has been developed in the early eighties in the plasma physics community [8,22,33-36]. A pioneering work for averaging nearly-periodic Hamiltonian systems has been given by Kruskal [31]. Up to now mathematically rigorous results for VA are lacking, which is surprising considering its importance for numerical plasma physics. In this work we shall close this mathematical gap and establish several cornerstones of variational GY-theory:

- existence of a GY-transformation leading to reduced dynamics;

- gauge-invariance;

- definition of a gyrokinetic equation; 
- strong error estimate for the gyrokinetic solution with respect to $f$, solution of (1).

We are able to prove existence with a new ansatz for the GY-transformation as a finite power series in $\varepsilon$, algebraic in the generating functions, in contrast to the usual Lie-transform approach, which relies on operator exponentials of Poisson brackets. Prerequisites for understanding existing formal VA-theories [9, 22, 23, 39, 44] include a firm knowledge about exterior calculus, differential forms and Lie transforms, with rare exceptions [34,41]. Our theory does not rely on these concepts and is thus more accessible for non-specualists. The long wavelength regime is considered, hence the inclusion of finiteLarmor radius effects postponed to a future work. We stress the non-uniqueness of transformations leading to GY-Lagrangians, which is overlooked in the existing VA-theories. A new GY-transformation is presented which leads to simpler equations of motion; this is possible due to the freedom of "unloading" complicated terms into the transformation (the generating functions), rather than keeping them in the Lagrangian.

The methodology of VA is carefully developed in this work. The concept of the "tangent map" between two coordinate representations of a manifolds's tangent bundle is introduced in detail. We then shift the focus to a particular class of Lagrangian functions of the form (13), linear in the tangent vectors. The VA-theory developed here could in principle be applied to a large class of highly-oscillatory problems, formulated in terms of this generic Lagrangian. The charged particle is a prototypical example and treated in detail.

Historically, the first approach towards reduced GY-models stems from averaging Newton's equation of motion for the charged particle [28,37]. Assuming a uniform static magnetic field, these can be solved exactly to yield the spiraling motion around the straight field lines. In this case the GY is well-defined and its trajectory follows a magnetic field line. Adding a static perpendicular electric field gives rise to a drift across field lines, but the GY is still well-defined. The problem complicates when the fields have curvature (non-homogeneous case). In this case several new drifts appear, for instance the curvature drift and the grad- $B$ drift [26]. On top of that, the GY is no longer well-defined: the center of the spiral cannot be computed in closed form, its location only approximated by an infinite series. In the non-homogeneous case the GY-dynamics are thus truncated dynamics (perturbation theory). For the validity of the theory it is thus essential to control the error that arises from truncation.

VA is based on a variational principle from which the equations of motion can be derived. Since the variational principle is coordinate independent it is particularly suited for averaging, which is nothing else than a change of coordinates, with a minimum amount of algebra. VA has the advantage that the Hamiltonian structure of the particle dynamics is not destroyed in the process. This leads in particular to conservation of a truncated energy and to conservation of a truncated phase space volume, which are easily identified. These and other conservation properties related to the Hamiltonian structure are beneficial for stability and accuracy of long-time numerical simulations.

Let us briefly mention some mathematical results on averaged particle dynamics in strong magnetic fields, not related to VA: Frénod and Sonnendrücker $[17,18]$ use two-scale convergence to establish limit models of the Vlasov-Poisson system in strong magnetic 
fields. The asymptotic behavior of the Vlasov-Maxwell system in strong magnetic fields has been considered by Bostan [3-6], relying on energy methods or averaging techniques. The transition from Vlasov to gyrokinetic equations has also been studied for example in $[15,19,25]$. Stroboscopic averaging is applied to the GY-problem in $[13,14]$. A WKBbased approach with emphasis on gyro-gauge has been presented in [42].

The article is organized as follows: in the preliminary section 2 we clarify some notation in 2.1 and introduce the equations of motion and their normalization in 2.2. In 2.3 we discuss the corresponding variational formulation; the notion of a Lagrangian function defined on the tangent bundle of the underlying manifold is presented in detail. We formulate the guiding-center problem as well as the full problem with electromagnetic external fields in the extended phase space. In section 2.4 we introduce the method of VA, which is based on the concept of the tangent map. Our new ansatz for the algebraic GYtransformation is stated here. The main results are collected in section 3, which is split into three subsections: in the preliminary part we define the guiding-center Lagrangian, equivalence of Lagrangians and the gyro-average operation and state the existence of solutions for the charge-particle dynamics in 3.1. Section 3.2 contains the existence results for the GY-transformation in Theorem 1. Explicit expressions for the GY-transformation, the corresponding GY-Hamiltonian and the generalized magnetic moment are given in section 4 . Finally, a gyrokinetic equation is defined in section 3.3; its strong solution is compared to the solution of the Vlasov equation in Theorem 2. Proofs that require a lot of algebra have been put into section 5 . We summarize the article and discuss future perspectives in section 6 .

\section{Preliminaries}

\section{$2.1 \quad$ Notation}

The vector product in $\mathbb{R}^{3}$ is denoted by ' $\times$ '. The symbol ' $\nabla$ ' denotes the usual gradient operator in $\mathbb{R}^{3}$, hence $\nabla=\left(\partial_{x_{1}}, \partial_{x_{2}}, \partial_{x_{3}}\right)^{\top}$. For a vector field $A: \mathbb{R}^{3} \rightarrow \mathbb{R}^{3}, A=\left(A_{1}, A_{2}, A_{3}\right)^{\top}$, we write $\nabla \times A$ to denote the curl-operator. Given a map $\tau: \mathbb{R}^{n} \rightarrow \mathbb{R}^{n}$, the Jacobian is denoted by $D \tau$, i.e. $(D \tau)_{i, j}=\partial \tau_{i} / \partial x_{j}$. For $n=3$ we denote the transpose Jacobian by

$$
\nabla A:=\left(\frac{\partial A_{j}}{\partial x_{i}}\right)_{1 \leq i, j \leq 3}=\left(\frac{\partial A}{\partial x}\right)^{\top}=(D A)^{\top} .
$$

The dot '.' denotes the scalar product in Euclidean space; it is also used to denote matrixvector multiplication in $\mathbb{R}^{n}$. For $b \in \mathbb{R}^{3}$ for example

$$
(b \cdot \nabla) A=b \cdot \nabla A=\nabla A^{\top} \cdot b .
$$




\subsection{Equations of motion and scaling}

Newton's equation of motion for a non-relativistic charged particle in an electromagnetic field can be written as

$$
\frac{\mathrm{d} x}{\mathrm{~d} t}=v, \quad \frac{\mathrm{d} v}{\mathrm{~d} t}=\frac{e}{m}[v \times B(x, t)+E(x, t)] .
$$

Here, $x$ stands for the particle position, $v$ its velocity, $e$ the particle's charge, $m$ its mass and $B$ and $E$ denote external magnetic and electric fields. The right-hand-side in the equation for $v$ is the Lorentz force, hence gravitational and other effects are neglected. Our first task is to formulate Newton's equation of motion in dimensionless form. For example, we write the solution $x$ as $x(t)=\hat{x} x^{\prime}\left(t^{\prime}\right)$, where $\hat{x}$ denotes the characteristic size (scale or unit) of the particle position and $x^{\prime}$ is a dimensionless function of $t^{\prime}=t / \hat{t}$, the time in units of $\hat{t}$. The characteristic size $\hat{x}$ could be for instance the diameter of our domain of study and $\hat{t}=\hat{\omega}^{-1}$, where $\hat{\omega}$ characterizes the frequency domain of interest. Similarly, $B(x, t)=\hat{B} B^{\prime}\left(x^{\prime}, t^{\prime}\right)$ for the fields. Hence,

$$
\frac{\mathrm{d} x^{\prime}}{\mathrm{d} t^{\prime}}=\frac{\hat{v}}{\hat{x} \hat{\omega}} v^{\prime}, \quad \frac{\mathrm{d} v^{\prime}}{\mathrm{d} t^{\prime}}=\frac{e \hat{B}}{m \hat{\omega}}\left[v^{\prime} \times B^{\prime}\left(x^{\prime}, t^{\prime}\right)+\frac{\hat{E}}{\hat{v} \hat{B}} E^{\prime}\left(x^{\prime}, t^{\prime}\right)\right] .
$$

The characteristic cyclotron frequency of the problem is $\hat{\omega}_{\mathrm{c}}=e \hat{B} / m$. We simplify via

$$
\begin{aligned}
& \text { a) } \hat{v}=\hat{x} \hat{\omega} \text {, } \\
& \text { b) } \varepsilon:=\frac{\hat{\omega}}{\hat{\omega}_{\mathrm{c}}} \text {, } \\
& \text { c) } \varepsilon_{\delta}:=\frac{\hat{E}}{\hat{v} \hat{B}} \text {. }
\end{aligned}
$$

In assumption a) we relate the velocity scale $\hat{v}$ to the chosen time- and space scales $\hat{\omega}^{-1}$ and $\hat{x}$. In b) we introduce a first parameter $\varepsilon$; if $\varepsilon \ll 1$ one enters the low-frequency regime, which means that the frequency of interest $\hat{\omega}$ is much smaller than the cyclotron frequency $\hat{\omega}_{\mathrm{c}}$. A second parameter $\varepsilon_{\delta}$ is introduced in c); it represents the ratio of the $E \times B$-velocity to the characteristic velocity $\hat{v}$. This parameter will also appear in the magnetic field, which we assume to be composed of two parts:

$$
B(x, t)=B_{0}(x)+\varepsilon_{\delta} B_{1}(x, t),
$$

a so-called "guide field" $B_{0}$, which is static and non-homogeneous and a dynamical part $B_{1}$ with amplitude $\varepsilon_{\delta}$. Thus $\varepsilon_{\delta}$ signifies the amplitude of the dynamical fields $E / v$ and $B_{1}$ with respect to the static guide field $B_{0}$. We introduce a third parameter $\varepsilon_{B}$ which measures the degree of inhomogeneity of the guide field $(\|\cdot\|$ is some matrix norm):

$$
\varepsilon_{B}:=\hat{x} \frac{\| \nabla B_{0}||}{\left|B_{0}\right|}
$$

Two cases of $\varepsilon_{B}$ shall be addressed in this paper: $\varepsilon_{B}=1$, which signifies that the guide field variations are on the scale $\hat{x}$, and $\varepsilon_{B}=\varepsilon$ which corresponds to less important variations of the guide field. 
Let us now insert the above definitions of $\varepsilon$-parameters into Newton's equations (3) and omit the primes to obtain

$$
\frac{\mathrm{d} x}{\mathrm{~d} t}=v, \quad \frac{\mathrm{d} v}{\mathrm{~d} t}=\frac{1}{\varepsilon} v \times\left[B_{0}\left(\varepsilon_{B} x\right)+\varepsilon_{\delta} B_{1}(x, t)\right]+\frac{\varepsilon_{\delta}}{\varepsilon} E(x, t) .
$$

Two orderings shall be addressed in this work:

$$
\text { 1) } \varepsilon_{\delta}=\varepsilon, \quad \varepsilon_{B}=1, \quad \text { 2) } \varepsilon_{\delta}=\varepsilon_{B}=\varepsilon
$$

Ordering 1) is rarely discussed in GY-theory, whereas case 2) is called the "maximal ordering" [9]. We point out that the ordering $\varepsilon_{B}=\varepsilon$ is implemented in all the aforementioned gyrokinetic models used for computer simulations, because of its relative simplicity with respect to the case $\varepsilon_{B}=1$ at the second order of expansion (see below).

\subsection{Variational formulation}

\subsubsection{Problem statement}

Under the scaling assumptions from the previous section, the initial-value problem (IVP) we consider reads

$$
\begin{cases}\frac{\mathrm{d} x}{\mathrm{~d} t}=v, & x\left(t_{0}\right)=x_{0}, \\ \frac{\mathrm{d} v}{\mathrm{~d} t}=\frac{v \times B_{0}\left(\varepsilon_{B} x\right)}{\varepsilon}+v \times B_{1}(x, t)+E(x, t), & v\left(t_{0}\right)=v_{0} .\end{cases}
$$

Here, we assume $x, x_{0} \in \Omega_{x} \subset \mathbb{R}^{3}, v, v_{0} \in \Omega_{v} \subset \mathbb{R}^{3}$ with $\operatorname{dim}\left(\Omega_{x}\right)=\operatorname{dim}\left(\Omega_{v}\right)=3$, $\Omega=\Omega_{x} \times \Omega_{v}$ open and bounded and $0<\varepsilon \leq \varepsilon_{\max }$. For $\varepsilon \ll 1$ system (9) represents a multi-scale problem with a fast, nearly-periodic motion around $B_{0}$. Classical averaging [40] can be applied to extract reduced dynamics free of the fast scale. However, system (9) is also rich in structure, a so-called Hamiltonian system. In order to see the structure we need to study its variational formulation.

\subsubsection{Lagrangian functions}

The variational formulation of (9) is based on a Lagrangian function, simply called the "Lagrangian". Lagrangians are defined on the tangent bundle of the underlying manifold, which in our study is the phase space, and map into the real numbers. We shall clarify this notion in more detail.

Let $M \subset \mathbb{R}^{n}$ denote an open subset of Euclidean space $\mathbb{R}^{n}$ with points $\mathfrak{m} \in M$, described by a single coordinate chart $\varphi: U \subset \mathbb{R}^{n} \rightarrow M, q \mapsto \mathfrak{m}$ ( $M$ is thus an $n$-dimensional differentiable manifold). $q$ are called coordinates of $M$ under the chart $\varphi$. The tangent space at point $\mathfrak{m} \in M$, denoted by $T M_{\mathfrak{m}}$, is the space af all vectors originating from $\mathfrak{m}$, hence $T M_{\mathfrak{m}}=\mathbb{R}^{n}$. More precisley $T M_{\mathfrak{m}}$ contains equivalence classes of curves through $\mathfrak{m}$, 
two curves being equivalent when they are tangent to each other at $\mathfrak{m}[1,2]$. We denote by $\xi \in T M_{\mathfrak{m}}$ an element of the tangent space at $\mathfrak{m}$.

Coordinates for $\xi \in T M_{\mathfrak{m}}$ can be constructed from the chart $\varphi$ as follows: for an open interval $I \subset \mathbb{R}$ let $c: I \rightarrow U$ denote a curve in the coordinate space $U$ with $c(0)=q$; then $\varphi(c)$ is a curve passing through $\mathfrak{m}$ at $t=0$ on the manifold $M$. The tangent at $t=0$ in the coordinate space is $\dot{q}:=\frac{\mathrm{d}}{\mathrm{d} t} c(0)$; in the tangent space $T M_{\mathfrak{m}}$ the tangent is

$$
\xi=\left.\frac{\mathrm{d} \varphi(c(t))}{\mathrm{d} t}\right|_{t=0}=\left.\sum_{j} \frac{\partial \varphi}{\partial q_{j}}\right|_{c(0)} \frac{\mathrm{d} c_{j}(0)}{\mathrm{d} t}=D \varphi(q) \cdot \dot{q} .
$$

Since this holds true for any curve $c$ passing through $q$ at $t=0$ we deduce that any $\xi \in T M_{\mathfrak{m}}$ can be written in the form $D \varphi(q) \cdot v$ for some $v \in \mathbb{R}^{n}$. It follows that the tangent space $T M_{\mathfrak{m}}$ is the image of the Jacobian $D \varphi(q)$; a basis of $T M_{\mathfrak{m}}$ is thus given by the columns of $D \varphi(q)$, which we denote by $\partial_{j}:=\frac{\partial \varphi}{\partial q_{j}}$ (covariant basis) [16]. The coefficients of a tangent vector $\xi$ in this basis are denoted by $\dot{q}$, hence

$$
\xi=D \varphi(q) \cdot \dot{q}=\sum_{j} \dot{q}_{j} \partial_{j}
$$

The union of all tangent spaces "attached" to $M$ at points $\mathfrak{m}$ is called the tangent bundle $T M$; its elements are tangent vectors. The chart $\varphi$ induces coordinates in the tangent bundle which we denote by $(q, \dot{q})$. The first coordinate $q \mapsto \mathfrak{m}$ identifies the tangent space and the second coordinate $\dot{q} \mapsto \xi$ identifies an element in that particular tangent space.

Another useful object is the dual to the tangent space $T M_{\mathfrak{m}}$, called the cotangent space $T^{*} M_{\mathfrak{m}}$. Its elements are covectors or linear forms $\gamma: T M_{\mathfrak{m}} \rightarrow \mathbb{R}$, mapping tangents into the real numbers. The chart $\varphi$ induces a basis also in the cotangent space: given the basis vectors $\partial_{j}$ of the tangent space, the dual basis $\mathrm{d}_{i} \in T^{*} M_{\mathfrak{m}}$ is defined by the property $\mathrm{d}_{i}\left(\partial_{j}\right)=\delta_{i j}$, where $\delta_{i j}$ is the Kronecker delta. Since for the Jacobains we have $D \varphi^{-1} D \varphi=I_{n}$ where $I_{n}$ is the identiy matrix, we deduce that the lines of $D \varphi^{-1}$ are the sought dual basis, thus $\mathrm{d}_{i}:=\nabla \varphi_{i}^{-1}$ (contravariant basis). Denoting the components of $\gamma$ in this basis by $\gamma_{i}$ we have, for general $\gamma \in T^{*} M_{\mathfrak{m}}$ and $\xi \in T M_{\mathfrak{m}}$,

$$
\gamma(\xi)=\sum_{i} \gamma_{i} \mathrm{~d}_{i}(\xi)=\sum_{i j} \gamma_{i} \dot{q}_{j} \mathrm{~d}_{i}\left(\partial_{j}\right)=\sum_{i j} \gamma_{i} \dot{q}_{j} \delta_{i j}=\gamma \cdot \dot{q}
$$

Hence the natural pairing between elements of the tangent space $T M_{\mathfrak{m}}$ and elements of its dual $T^{*} M_{\mathfrak{m}}$ can be written as a scalar product in $\mathbb{R}^{n}$ with respect to the bases induced by the chart $\varphi$. We shall use this convenient notation throughout this work.

The union of all cotangent spaces at points $\mathfrak{m} \in M$ is called the cotangent bundle and denoted by $T^{*} M$. The chart $\varphi$ induces coordinates in the cotangent bundle: an element is identified via $\gamma(q)$, where $q \mapsto \mathfrak{m}$ identifies the cotangent space (dual to the tangent space at $\mathfrak{m}$ ) and the "vector" $\gamma$ holds the components of the linear form in that particular cotangent space, such that the duality pairing can be written as the scalar product (12).

We are now equipped to define a Lagrangian function on the tangent bundle of the manifold $M$. We shall consider dynamical systems defined by a particular class of Lagrangians 
$L: T M \rightarrow \mathbb{R}$ which, in local coordinates $(q, \dot{q})$ defined by some chart $\varphi: U \subset \mathbb{R}^{n} \rightarrow M$, can be written as

$$
L(q, \dot{q})=\gamma(q) \cdot \dot{q}-H(q) .
$$

Here, $H: M \rightarrow \mathbb{R}$ is called the Hamiltonian and $\gamma \in T^{*} M$ is the symplectic form, in the sense of (12). We will now discuss how the charged-particle problem (9) can be deduced from such a Lagrangian by a variational principle.

\subsubsection{The action principle}

Given the Lagrangian (13) the dynamics follow from a variational principle on curves in the coordinate space $U$. Let us denote such curves by $q(s)$, or more precisely by $q: I \rightarrow U$ for some open interval $I \subset \mathbb{R}$. Let us further define the following functional on the space of curves,

$$
\mathcal{A}[q]:=\int_{I} L\left(q(s), \frac{\mathrm{d}}{\mathrm{d} s} q(s)\right) d s .
$$

The variational (action) principle $\delta \mathcal{A} / \delta q=0$ yields the Euler-Lagrange equations

$$
\frac{\partial L}{\partial q}-\frac{\mathrm{d}}{\mathrm{d} s} \frac{\partial L}{\partial \dot{q}}=0
$$

which, for $L$ given by (13), become

$$
\omega \cdot \frac{\mathrm{d} q}{\mathrm{~d} s}=\frac{\partial H}{\partial q},
$$

where $\omega:=(D \gamma)^{T}-D \gamma$ is called the Lagrange matrix. We assume that $\omega$ is invertible on $U$ and write $J:=\omega^{-1}$. Then system (16) can be written as

$$
\frac{\mathrm{d} q}{\mathrm{~d} s}=\{q, H\},
$$

where $\{G, H\}:=\partial G / \partial q \cdot J \cdot \partial H / \partial q$ denotes the Poisson bracket, defined for differentiable functions $G, H: U \rightarrow \mathbb{R}$. The bracket is bilinear, anti-symmetric and satisfies the Jacobi identity

$$
\{F,\{G, H\}\}+\{H,\{F, G\}\}+\{G,\{H, F\}\}=0 .
$$

Systems of the form (16) where $\omega$ is invertible are called non-canonical symplectic systems, which belong to the larger class of Hamiltonian systems. It is an immediate consequence of (17) that $H(q)$ is a constant of the motion, $\frac{\mathrm{d}}{\mathrm{d} s} H=0$. Moreover, it can be shown that the flow of (17) conserves the phase space volume $\sqrt{\operatorname{det} \omega}$, computed from the determinant of the Lagrange matrix $\omega$. Other constants of the motion are the so-called Casimirs and the momentum maps [1,2]. Exact conservation of these invariants on the discrete level leads to improved long-time stability and accuracy of numerical schemes. An example of such a symplectic integrator is the well-known Störmer-Verlet scheme [24]. 


\subsubsection{The guiding-center problem}

If the dynamical fields $E$ and $B_{1}$ in (9) are zero, the problem of averaging reduces to the so-called guiding-center (GC) problem. In this case the system (9) is autonomous and a Lagrangian of the generic form (13) can be formulated in the coordinate space $U=\Omega$; it reads

$$
L_{\mathrm{a}}=\left(v+\frac{A_{0}(x)}{\varepsilon}\right) \cdot \dot{x}-\frac{|v|^{2}}{2} .
$$

Here, $A_{0}$ is the vector potential related to the guide field via $B_{0}=\nabla \times A_{0}$. In terms of the generic form (13) we have

$$
\gamma=\gamma_{\mathrm{a}}=\left(v+\frac{A_{0}(x)}{\varepsilon}, 0,0,0\right), \quad H=H_{\mathrm{a}}=\frac{|v|^{2}}{2} .
$$

The velocity components of the symplectic form are zero. It can be easily checked that the Euler-Lagrange equations corresponding to $L_{\mathrm{a}}$ yield the equations (9) without dynamical fields. Moreover, we know that this system is non-canonical symplectic because its Lagrange matrix is invertible. The kinetic energy $H_{\mathrm{a}}$ is conserved during the motion.

Variational averaging of the Lagrangian (18) has been studied extensively on the formal level; the first rigorous results are presented in this work. A review can be found in [12]. Higher-order computations of the asymptotic GC-expansion have recently been reported $[10,43]$. The computations in this paper will reproduce the standard GC-results up to second order in the GC-Hamiltonian and GC-symplectic form. The first-order GCLagrangian is defined in (41).

\subsubsection{Full problem with dynamical fields}

In case that the dynamical fields $E$ and/or $B_{1}$ are not zero the system (9) is non-autonomous. It becomes an autonomous system in the extended coordinate space $U=\Omega \times \mathbb{R}^{2}$ with coordinates $q=(x, v, t, w)$. Here, the time $t$ and the energy $w$ are dependent variables and the independent variable is denoted by $s$. Symplectic form and Hamiltonian are introduced as

$$
\gamma_{\mathrm{ext}}:=\left(\gamma_{\mathrm{a}}, 0,0\right)+\left(A_{1}(x, t), 0,0,0,-w, 0\right), \quad H_{\mathrm{ext}}:=H_{\mathrm{a}}+\phi(x, t)-w .
$$

Here, the dynamical electromagnetic potentials $A_{1}$ and $\phi$ are such that

$$
B_{1}=\nabla \times A_{1}, \quad E=-\nabla \phi-\frac{\partial A_{1}}{\partial t} .
$$

The Lagrangian is of the generic form (13) and reads

$$
L=\left(v+\frac{A_{0}(x)}{\varepsilon}+A_{1}(x, t)\right) \cdot \dot{x}-w \dot{t}-\frac{|v|^{2}}{2}-\phi(x, t)+w .
$$

The corresponding Lagrange matrix is invertible and the system is non-canonical symplectic with conserved energy $H_{\text {ext }}$. The Euler-Lagrange equation for $w$ automatically 
yields $\frac{\mathrm{d}}{\mathrm{d} s} t=1$ and thus $t=s$. The charged-particle dynamics are found to occur on the hyper-surface $H_{\text {ext }}=0$ of the extended coordinate space. For simplicity during variational averaging we directly impose $H_{\text {ext }}=0$ which means $w=|v|^{2} / 2+\phi=: H$; this leads to the "extended Lagrangian" [2]

$$
L_{\mathrm{I}}:=\left.L\right|_{w=H}=\left(v+\frac{A_{0}(x)}{\varepsilon}+A_{1}(x, t)\right) \cdot \dot{x}-H \dot{t},
$$

where the coordinate space is $\Omega_{\mathrm{I}}:=\Omega \times \mathbb{R}$ with elements $q=(x, v, t)$. The Lagrangian (23) is written as $L_{\mathrm{I}}(q, \dot{q})=\gamma_{\mathrm{I}}(q) \cdot \dot{q}$, where

$$
\gamma_{\mathrm{I}}:=\left(v+\frac{A_{0}(x)}{\varepsilon}+A_{1}(x, t), 0,0,0,-H\right)
$$

is the well-known Poincaré-Cartan form; it is the starting point for any gyro-averaging theory in the variational framework.

\subsection{Change of coordinates}

\subsubsection{What is variational averaging?}

The aim of variational GY-theory is to preserve the symplectic structure of the chargedparticle dynamics (9), manifested by a Poisson bracket (17), when averaging the fast scale due to the $v \times B_{0}$ motion. The structure originates from the generic form of the Lagrangian (22). Hence, averaging directly on the level of the Lagrangian while keeping the generic form (13) is the favorable strategy, as outlined in [34]. "Averaging" in this context can be defined by the following steps:

1. Identify a fast variable, the gyro-angle, that changes on the time scale $\varepsilon$ due to the $v \times B_{0}$ motion. This is done by a "preliminary map" in the extended Lagrangian (23).

2. Find a change of coordinates in phase space that decouples the fast motion of the gyro-angle from the remaining equations on the slow scale. Suppose $\alpha$ denotes the fast variable, then the coordinate map should eliminate $\alpha$ from the Lagrangian at successive orders in $\varepsilon$, up to the desired order $\varepsilon^{N}$.

3. The "decoupling" is then accomplished by truncating the new Lagrangian at order $N$, which means neglecting terms of order $\varepsilon^{N+1}$ :

$$
L^{\varepsilon}=\underbrace{\frac{L_{-1}}{\varepsilon}+L_{0}+\varepsilon L_{1}+\ldots+\varepsilon^{N} L_{N}}_{=: L_{\mathrm{gy}}^{(N)}}+\varepsilon^{N+1} L_{N+1}+\ldots
$$

4. The "decoupled" equations of motion are the Euler-Lagrange equations stemming from the truncated Lagrangian $L_{\mathrm{gy}}^{(N)}$. They feature the slow variables which, by construction, can be computed independently of the fast variable $\alpha$. The term "averaged 
dynamics" refers to the dynamics of the slow variables. Moreover, the Euler-Lagrange equation (15) for $\alpha$ yields

$$
\frac{\mathrm{d}}{\mathrm{d} s} \frac{\partial L_{\mathrm{gy}}^{(N)}}{\partial \dot{\alpha}}=0
$$

which states the conservation of the "generalized magnetic moment" $\widehat{\mu}:=\partial L_{\mathrm{gy}}^{(N)} / \partial \dot{\alpha}$. We identify three fundamental questions related to the above approach:

- Under what premise does a coordinate map leading to (25) exist?

- How does the truncation error in the Lagrangian translate to errors in the equations of motion?

- In what way can the averaged equations be used to derive a gyrokinetic equation?

These questions, among others, shall be addressed in the course of this work. The main tool for variational averaging is the "tangent map", which allows us to transform Lagrangians defined on tangent bundles; it is introduced next.

\subsubsection{The tangent map}

Let $M \subset \mathbb{R}^{n}$ with $\mathfrak{m} \in M$ denote the manifold introduced in section 2.3.2, described by the single coordinate chart $\varphi: U \subset \mathbb{R}^{n} \rightarrow M, q \mapsto \mathfrak{m}$. Suppose $\psi: V \subset \mathbb{R}^{n} \rightarrow M$ stands for a different chart describing the same manifold $M$ in the coordinates $l \mapsto \mathfrak{m}$. Then the map $\tau: V \rightarrow U, l \mapsto q$ given by $q=\tau(l)=\varphi^{-1} \circ \psi(l)$ defines a change of coordinates on the manifold $M$. The map $\tau$ is one-to-one and differentiable with differentiable inverse, hence a diffeomorphism. Its Jacobian is $D \tau=D \varphi^{-1} D \psi$.

The transformation law for elements $\xi$ of the tangent space $T M_{\mathfrak{m}}$ is straightforward: from (11) we have

$$
\xi=D \varphi(q) \cdot \dot{q}=D \psi(l) \cdot \dot{l} .
$$

The components $\dot{q}$ can thus be expressed in terms of the components $i$ via

$$
\dot{q}=D \varphi(\tau(l))^{-1} D \psi(l) \cdot \dot{l}=D \tau(l) \cdot \dot{l} .
$$

Definition 1. (Tangent map.) Given a change of coordinates $\tau: l \mapsto q$ on the manifold $M$, the associated "tangent map" $T \tau:(l, \dot{l}) \mapsto(q, \dot{q})$ relating two coordinate systems of the tangent bundle $T M$ is defined by

$$
(q, \dot{q})=T \tau(l, i):=(\tau(l), D \tau(l) \cdot \dot{l}) .
$$

The tangent map is the principal tool for the theory of variational averaging presented in this work. It will be used to transform the extended Lagrangian (23) from the coordinates $q \in \Omega_{\mathrm{I}}$ to new coordinates $l \in V$ :

$$
L_{\mathrm{I}}(q, \dot{q})=\gamma_{\mathrm{I}}(q) \cdot \dot{q}=\underbrace{\gamma_{\mathrm{I}}(\tau(l)) \cdot D \tau(l)}_{=: \widehat{\gamma}_{\mathrm{I}}(l)} \cdot \dot{l}=\widehat{\gamma}_{\mathrm{I}}(l) \cdot \dot{l} .
$$


Here, we almost accidentally uncovered the transformation law of covectors (elements of the cotangent space), $\gamma_{\mathrm{I}} \circ \tau=D \tau^{-T} \widehat{\gamma}_{\mathrm{I}}$. Variational averaging is built on the fact that in (30) the generic form of the extended Lagrangian $L_{\mathrm{I}}$ is preserved under the tangent map. Moreover, from the transformation law of cotangents we can deduce that the new Lagrange matrix $\widehat{\omega}=\left(D \widehat{\gamma}_{\mathrm{I}}\right)^{T}-D \widehat{\gamma}_{\mathrm{I}}$ is invertible, and hence the symplectic structure preserved.

\subsubsection{Preliminary transformation}

We apply a preliminary coordinate map to the extended Lagrangian $L_{\mathrm{I}}: T \Omega_{\mathrm{I}} \rightarrow \mathbb{R}$ from (23) for the purpose of identifying the fast variable (gyro-angle), which is then subjected to averaging. We start from a local, orthonormal basis $\left(e_{1}(x), e_{2}(x), b_{0}(x)\right)$ that satisfies $b_{0}=e_{1} \times e_{2}, e_{1}=e_{2} \times b_{0}, e_{2}=b_{0} \times e_{1}$ such that $b_{0} \cdot e_{1} \times e_{2}=1$ and the basis is right-handed. New velocity coordinates are introduced as

$$
\begin{aligned}
v_{\|} & :=v \cdot b_{0}(x), \\
v_{\perp} & :=\left|b_{0}(x) \times v \times b_{0}(x)\right|=\left|v-v \cdot b_{0}(x) b_{0}(x)\right|, \\
\theta & :=-\arctan 2\left(\frac{v \cdot e_{2}(x)}{v \cdot e_{1}(x)}\right),
\end{aligned}
$$

such that $v=v_{\|} b_{0}+v_{\perp} c_{0}$, where $c_{0}:=e_{1}(x) \cos \theta-e_{2}(x) \sin \theta$. Together with the unit vector $a_{0}:=e_{1}(x) \sin \theta+e_{2}(x) \cos \theta$, the triple $\left(a_{0}, b_{0}, c_{0}\right)$ is an orthonormal basis of $\Omega_{v}$ at each $x \in \Omega_{x}$. Moreover, one has the identities

$$
b_{0} \times v=v_{\perp} a_{0}, \quad b_{0} \times v \times b_{0}=v_{\perp} c_{0} .
$$

Now let $\Omega_{\mathrm{I}}^{\prime}$ denote the extended phase space with velocity coordinates (31), i.e. for $q^{\prime} \in \Omega_{\mathrm{I}}^{\prime}$ we have $q^{\prime}=\left(x, v_{\|}, v_{\perp}, \theta, t\right)$. The preliminary map is thus

$$
\tau^{\prime}: \Omega_{\mathrm{I}}^{\prime} \rightarrow \Omega_{\mathrm{I}}, \quad q^{\prime} \mapsto q
$$

defined by

$$
x=x, \quad v=v_{\|} b_{0}(x)+v_{\perp} c_{0}(x, \theta), \quad t=t,
$$

with Jacobian determinant $-v_{\perp}$. The transformed Lagrangian $L^{\prime}$ is obtained from (23) by inserting (34),

$$
L^{\prime}\left(q^{\prime}, \dot{q}^{\prime}\right)=\left[v_{\|} b_{0}(x)+v_{\perp} c_{0}(x, \theta)+\frac{A_{0}(x)}{\varepsilon}+A_{1}(x, t)\right] \cdot \dot{x}-\left[\frac{v_{\|}^{2}}{2}+\frac{v_{\perp}^{2}}{2}+\phi(x, t)\right] \dot{t},
$$

It is straightforward to show from the Euler-Lagrange equations

$$
\frac{\partial L^{\prime}}{\partial q^{\prime}}-\frac{\mathrm{d}}{\mathrm{d} s} \frac{\partial L^{\prime}}{\partial \dot{q}^{\prime}}=0
$$

that $\theta$ is the fast gyro-angle, changing on the time scale $\varepsilon$. 


\subsubsection{Algebraic GY-transformations}

The second step of variational averaging requires a coordinate map $\tau^{\varepsilon}: \Omega_{\mathrm{gy}} \rightarrow \Omega_{\mathrm{I}}^{\prime}, q_{\mathrm{gy}} \mapsto q^{\prime}$ which eliminates the fast variable $\alpha \mapsto \theta$ from the Lagrangian (35), order by order in $\varepsilon$. The second transformation is thus assumed to be a finite power series in $\varepsilon$, defined by

$$
q^{\prime}=\tau^{\varepsilon}\left(q_{\mathrm{gy}}\right):=q_{\mathrm{gy}}+\sum_{n=1}^{N+1} \varepsilon^{n} \mathbf{G}_{n}\left(q_{\mathrm{gy}}\right),
$$

where $N \geq 0$ denotes the order of the transformation and the $\mathbf{G}_{n}: \Omega_{\mathrm{gy}} \rightarrow \Omega_{\mathrm{I}}^{\prime}$ are smooth maps, the so-called "generating functions" or generators of the transformation. They should be bounded uniformly in $\varepsilon$, such that $\lim _{\varepsilon \rightarrow 0} \tau^{\varepsilon}=\tau^{0}$ is the identity. Note that one needs $N+1$ generators in the $N$-th order transformation and that these generators occur merely as coefficients in the $\varepsilon$-series (algebraic dependence on the generators). The GY-coordinates $q_{\mathrm{gy}}=\left(q_{\mathrm{gy}, i}\right)_{1 \leq i \leq 7}$ and the generators $\mathbf{G}_{n}=\left(G_{n, i}\right)_{1 \leq i \leq 7}$ are denoted by

$$
\begin{aligned}
& \left(q_{\mathrm{gy}, i}\right)_{1 \leq i \leq 3}=r, \quad q_{\mathrm{gy}, 4}=q_{\|}, \quad q_{\mathrm{gy}, 5}=q_{\perp}, \quad q_{\mathrm{gy}, 6}=\alpha, \quad q_{\mathrm{gy}, 7}=t \\
& \left(G_{n, i}\right)_{1 \leq i \leq 3}=\varrho_{n} \quad G_{n, 4}=G_{n}^{\|}, \quad G_{n, 5}=G_{n}^{\perp}, \quad G_{n, 6}=G_{n}^{\theta}, \quad G_{n, 7}=0,
\end{aligned}
$$

where $r \in \mathbb{R}^{3}$ denotes the GY-position, $q_{\|}$and $q_{\perp}$ are the respective parallel and perpendicular GY-velocities and $\alpha$ stands for the gyro-angle. The time coordinate $t$ rests untransformed since we assume its generators to be zero at all orders. Component-wise, the transformation (37) thus reads

$$
\begin{aligned}
x & =r+\sum_{n=1}^{N+1} \varepsilon^{n} \varrho_{n}\left(q_{\mathrm{gy}}\right), \\
v_{\|} & =q_{\|}+\sum_{n=1}^{N+1} \varepsilon^{n} G_{n}^{\|}\left(q_{\mathrm{gy}}\right), \\
v_{\perp} & =q_{\perp}+\sum_{n=1}^{N+1} \varepsilon^{n} G_{n}^{\perp}\left(q_{\mathrm{gy}}\right), \\
\theta & =\alpha+\sum_{n=1}^{N+1} \varepsilon^{n} G_{n}^{\theta}\left(q_{\mathrm{gy}}\right), \\
t & =t .
\end{aligned}
$$

Moreover, from the definition of the tangent map one obtains

$$
\dot{x}=\dot{r}+\sum_{n=1}^{N+1} \varepsilon^{n} \dot{\varrho}_{n}\left(q_{\mathrm{gy}}, \dot{q}_{\mathrm{gy}}\right), \quad \dot{\varrho}_{n}:=\frac{\partial \varrho_{n}}{\partial q_{\mathrm{gy}}} \cdot \dot{q}_{\mathrm{gy}} .
$$

Starting from (35) the tangent map leads to the extended Lagrangian $L^{\varepsilon}$ in the variables $q_{\mathrm{gy}}$,

$$
\left.L^{\varepsilon}\left(q_{\mathrm{gy}}, \dot{q}_{\mathrm{gy}}\right):=L^{\prime}\left(T \tau^{\varepsilon}\left(q_{\mathrm{gy}}, \dot{q}_{\mathrm{gy}}\right)\right)=L^{\prime}\left(\tau^{\varepsilon}\left(q_{\mathrm{gy}}\right), D \tau^{\varepsilon}\left(q_{\mathrm{gy}}\right) \cdot \dot{q}_{\mathrm{gy}}\right)\right) .
$$


If we assume sufficiently regular potentials $A$ and $\phi$, the definition of $\tau^{\varepsilon}$ as a power series in (37) translates to a Taylor expansion of $L^{\prime}$ around $\left(q_{\mathrm{gy}}, \dot{q}_{\mathrm{gy}}\right)$, leading to a representation of $L^{\varepsilon}$ in the form (25). The generators $\mathbf{G}_{n}$ are still undetermined in this formulation. As outlined by Kruskal and Littlejohn [31,34], they can be chosen order by order such that the truncated Lagrangian $L_{\mathrm{gy}}^{(N)}$ is independent of the GY-angle $\alpha$.

Remark 1. The gyro-transformation (GT) that leads to the Lagrangian (25) will be composed of two transformations, $\tau_{\text {gy }}^{\varepsilon}=\tau^{\prime} \circ \tau^{\varepsilon}$, where $\tau^{\prime}$ is the "preliminary" transformation independent of $\varepsilon$ and $\tau^{\varepsilon}$ denotes the algebraic GY-transformation (37). Even though $\tau_{\text {gy }}^{\varepsilon}$ is a composition, it must not be confused with the "two-step" GT [9], where only the static $B_{0}$ is considered at first (guiding-center problem) and only after the dynamical fields $B_{1}$ and $E$ are taken into account. Indeed, the two-step GT is really a three-step GT since the preliminary transformation $\tau^{\prime}$ is applied also in this case. Our procedure corresponds to what is known as the "one-step" GT.

\section{Main results}

\subsection{Preliminaries}

The main results have been arranged into three categories which are presented in the following three subsections: section 3.1 contains a preliminary result on the existence of solutions to the initial value problem (9), under the here used regularity assumptions on the electromagnetic potentials:

Assumption 1. For $N \geq 0$ we suppose $A_{0} \in C^{N+3}\left(\Omega_{x}\right), A_{1} \in C^{N+2}\left(\Omega_{x} \times \mathbb{R}\right)$ for the vector potential and $\phi \in C^{N+1}\left(\mathbb{R} ; C^{N+2}\left(\Omega_{x}\right)\right)$ for the electrostatic potential.

In section 3.2 the first main result Theorem 1 shows that this regularity is sufficient for the existence of a truncated Lagrangian $L_{\mathrm{gy}}^{(N)}$ in (25), independent of the gyro-angle, for arbitrary order $N$. Finally, section 3.3 concerns the error in the averaged dynamics due to truncation of the true Lagrangian. We give an exact definition of a gyrokinetic equation and compute a strong error bound for its solution with respect to the solution of the Vlasov equation (1) in our second main result, Theorem 2. Let us start with some useful definitions:

Definition 2. (Guiding-center Lagrangian.) The guiding-center (GC) Lagrangian is defined as

$$
L_{\mathrm{gc}}\left(q_{\mathrm{gy}}, \dot{q}_{\mathrm{gy}}\right):=\left[q_{\|} b_{0}(r)+\frac{A_{0}(r)}{\varepsilon}\right] \cdot \dot{r}+\varepsilon \frac{q_{\perp}^{2}}{2\left|B_{0}(r)\right|} \dot{\alpha}-\left[\frac{q_{\|}^{2}}{2}+\frac{q_{\perp}^{2}}{2}\right] \dot{t} .
$$

Definition 3. (Equivalence of Lagrangians.) Two Lagrangians $L^{*}, L$ defined on $T M$ are equivalent, $L^{*} \sim L$, if there exists a function $S: M \rightarrow \mathbb{R}$ such that $L^{*}=L+\partial S / \partial q \cdot \dot{q}$ in some coordinates $q$. Equivalent Lagrangians lead to the same Euler-Lagrange equations. 
Definition 4. (Gyro-average.) The gyro-average and fluctuations of a function $G$ that is $2 \pi$-periodic in $\alpha$ are defined by

$$
\langle G\rangle(\cdot):=\frac{1}{2 \pi} \int_{0}^{2 \pi} G(\cdot, \alpha) d \alpha, \quad \widetilde{G}:=G-\langle G\rangle .
$$

Assumption 2. In the initial value problem (9) we denote by $\rho$ the radius of the largest ball in $\Omega_{x}$ containing $x_{0}$, that is $\rho:=\sup _{R \in \mathbb{R}}\left(\left\{x \in \mathbb{R}^{3}:\left|x-x_{0}\right|<R, x \in \Omega_{x}\right\}\right)$. Moreover, the velocity space is bounded by a maximal kinetic energy, $\Omega_{v}=\left\{v \in \mathbb{R}^{3}:|v|^{2}<v_{\max }^{2}\right\}$, and $\rho_{\text {kin }}:=\left(v_{\max }^{2}-\left|v_{0}\right|^{2}\right) / 2$.

Lemma 1. Let $\varepsilon>0$. Under the assumptions 1 and 2 the initial value problem (9) has a unique solution which exists for $t \in\left[t_{0}, t_{0}+T\right]$ with $T=\min \left(\rho / v_{\max }, \rho_{\mathrm{kin}} /\left(E_{\max } v_{\max }\right)\right)$, independent of $\varepsilon$.

Proof. Due to assumption 1 the fields in (9) are continuous on a bounded domain $\Omega$; hence the electric field has an upper bound, $|E|<E_{\max }$. We only need to check at which time the solution will leave $\Omega$. Integrating the first equation and taking the norm yields

$$
\left|x(t)-x_{0}\right| \leq\left(t-t_{0}\right) v_{\max }<\rho \quad \Longrightarrow \quad\left(t-t_{0}\right)<\frac{\rho}{v_{\max }} .
$$

Multiplying the second equation by $v$ and integrating in time leads to

$$
\frac{1}{2}\left(|v(t)|^{2}-\left|v_{0}\right|^{2}\right) \leq\left(t-t_{0}\right) E_{\max } v_{\max }<\rho_{\text {kin }} \quad \Longrightarrow \quad\left(t-t_{0}\right)<\frac{\rho_{\text {kin }}}{E_{\max } v_{\max }},
$$

which determines the time interval $T$.

\subsection{Existence of an algebraic GY-map $\tau^{\varepsilon}$}

Proposition 1. (Series-expansion of $L^{\varepsilon}$.) Under assumption 1 the Lagrangian $L^{\varepsilon}$ in (40) is equivalent to the series expansion

$$
L^{\varepsilon} \sim \frac{1}{\varepsilon} L_{-1}+L_{0}+\sum_{n=1}^{N} \varepsilon^{n} L_{n}+O\left(\varepsilon^{N+1}\right),
$$

with the terms

$$
\begin{aligned}
L_{-1}= & A_{0} \cdot \dot{r} \\
L_{0}= & \left(q_{\|} b_{0}+q_{\perp} c_{0}-\varrho_{1} \times B_{0}+A_{1}\right) \cdot \dot{r}-\left(\frac{q_{\|}^{2}}{2}+\frac{q_{\perp}^{2}}{2}+\phi\right) \dot{t}, \\
L_{1 \leq n \leq N}= & {\left[G_{n}^{\|} b_{0}+G_{n}^{\perp} c_{0}-\varrho_{n+1} \times B_{0}-\varrho_{n} \times B_{1}+\mathcal{Q}_{n}\right] \cdot \dot{r} } \\
& -\left(q_{\|} G_{n}^{\|}+q_{\perp} G_{n}^{\perp}-\varrho_{n} \cdot E\right) \dot{t}+\mathcal{L}_{n} .
\end{aligned}
$$

Here, the linear form $\mathcal{Q}_{n}$ and the Lagrangian $\mathcal{L}_{n}$ are given in (100) and (101), respectively. For $n=1$ and $n=2$ they can be written in terms of the fields $B_{0}, B_{1}$ and $E$ (gaugeinvariance). 
Proof. The proof is written in section 5.1.

Theorem 1. (Existence of $\tau^{\varepsilon}$.) Under assumption 1, for all $N \geq 1$, there exist generators $\mathbf{G}_{n} \in C^{2}\left(\Omega_{\mathrm{gy}}\right), 1 \leq n \leq N+1$, of the algebraic $G Y$-transformation $\tau^{\varepsilon}$ such that $L^{\varepsilon}$ from (43) is equivalent to $L^{\varepsilon} \sim L_{\mathrm{gy}}^{(N)}+O\left(\varepsilon^{N+1}\right)$, where the $G Y$-Lagrangian reads

$$
L_{\mathrm{gy}}^{(N)}=L_{\mathrm{gc}}+A_{1} \cdot \dot{r}-\phi \dot{t}+\varepsilon^{2} \delta \mu^{(N)} \dot{\alpha} .
$$

Here, $L_{\mathrm{gc}}$ denotes the guiding-center Lagrangian defined in $(41)$ and $\delta \mu^{(N)}: \Omega_{\mathrm{gy}} \rightarrow \mathbb{R}$ is the $N$-th order correction to the magnetic moment $\mu=q_{\perp}^{2} /\left(2\left|B_{0}\right|\right)$, independent of $\alpha$.

Proof. The proof is written in section 5.2.

Remark 2. The existence result from Theorem 1 does not imply that the transformation $\tau^{\varepsilon}$ exists as $N \rightarrow \infty$, because we cannot say that the series (37) converges in this limit. Convergence of the series would mean that a gyro-center of the motion exists globally. This is true for the constant field case $B=$ const. and $E=$ const. but it is not clear in the non-homogeneous case. In practice, however, only low orders $N \leq 2$ are important for numerical purposes.

\subsection{An error estimate for gyrokinetics}

Due to the Euler-Lagrange equation (26), the result in Theorem 1 leads to the conservation of the generalized magnetic moment $\widehat{\mu}$ during the GY-motion, where

$$
\widehat{\mu}:=\frac{q_{\perp}^{2}}{2\left|B_{0}\right|}+\varepsilon \delta \mu^{(N)} .
$$

In order to reduce the dimension of the problem, $\widehat{\mu}$ must be adopted as one of the coordinates. In particular, we shall assume that there is a one-to-one correspondence $\widehat{\tau}: \widehat{\mu} \mapsto q_{\perp}$, which is the case in all of the results presented in section 4. The full GY-transformation from $x$ - $v$ - $t$-coordinates (extended phase space $\Omega_{\mathrm{I}}$ ) to the GY-coordinates $\widehat{q}_{\mathrm{gy}} \in \widehat{\Omega}_{\mathrm{gy}}$ with generalized magnetic moment, hence $\widehat{q}_{\mathrm{gy}}=\left(r, q_{\|}, \widehat{\mu}, \alpha, t\right)$, is given by the composition

$$
\tau_{\mathrm{gy}}^{\varepsilon}: \widehat{\Omega}_{\mathrm{gy}} \rightarrow \Omega_{\mathrm{I}}, \quad \tau_{\mathrm{gy}}^{\varepsilon}=\tau^{\prime} \circ \tau^{\varepsilon} \circ \widehat{\tau} .
$$

It follows from Theorem 1 that the exact dynamics can be obtained from the Lagrangian

$$
L^{\varepsilon} \sim L_{\mathrm{gy}}^{(N)}+O\left(\varepsilon^{N+1}\right)
$$

which is now written in terms of the coordinates $\widehat{q}_{\mathrm{gy}}$ as

$$
L_{\mathrm{gy}}^{(N)}=\frac{1}{\varepsilon} A^{*} \cdot \dot{r}-H_{\mathrm{gy}} \dot{t}+\varepsilon \widehat{\mu} \dot{\alpha},
$$


with the auxiliary potential $A^{*}$ and the GY-Hamiltonian $H_{\text {gy }}$ defined by

$$
\begin{aligned}
A^{*} & :=A_{0}+\varepsilon A_{1}+\varepsilon q_{\|} b_{0}, \\
H_{\mathrm{gy}} & :=\frac{q_{\|}^{2}}{2}+\widehat{\mu}\left|B_{0}\right|+\phi+\varepsilon \delta H^{(N)} .
\end{aligned}
$$

Here, the Hamiltonian correction $\delta H^{(N)}$ stems from the transformation of the term $q_{\perp}^{2} / 2$ under the map $\widehat{\tau}$. It is a remarkable feature that $\delta H^{(N)}$ is the only term in the Lagrangian that changes with the order $N$ of the transformation. This means in particular that, in the coordinates $\widehat{q}_{\mathrm{gy}}$, the non-time components $\left(A^{*} / \varepsilon, 0,0, \varepsilon \widehat{\mu}\right)$ of the GY-symplectic form are the same for all $N$.

Setting $\dot{t}=1$ in (48), a straightforward computation yields the Euler-Lagrange equations of (47), here stated with initial conditions, and with the abbreviations $B^{*}=\nabla \times A^{*}$, $B_{\|}^{*}=B^{*} \cdot b_{0}$ and $E^{*}=E-\widehat{\mu} \nabla\left|B_{0}\right|-\varepsilon \nabla \delta H^{(N)}$,

$$
\left(P^{\varepsilon}\right)\left\{\begin{array}{lll}
\frac{\mathrm{d} r}{\mathrm{~d} t}=\frac{1}{B_{\|}^{*}}\left(q_{\|}+\varepsilon \frac{\partial \delta H^{(N)}}{\partial q_{\|}}\right) B^{*}+\varepsilon \frac{1}{B_{\|}^{*}} E^{*} \times b_{0}+O\left(\varepsilon^{N+2}\right), & r\left(t_{0}\right)=r_{0}, \\
\frac{\mathrm{d} q_{\|}}{\mathrm{d} t}=\frac{1}{B_{\|}^{*}} B^{*} \cdot E^{*}+O\left(\varepsilon^{N+1}\right), & q_{\|}\left(t_{0}\right)=q_{\| 0} \\
\frac{\mathrm{d} \widehat{\mu}}{\mathrm{d} t}=O\left(\varepsilon^{N}\right), & \widehat{\mu}\left(t_{0}\right)=\widehat{\mu}_{0}, \\
\frac{\mathrm{d} \alpha}{\mathrm{d} t}=\frac{\left|B_{0}\right|}{\varepsilon}+\frac{\partial \delta H^{(N)}}{\partial \widehat{\mu}}+O\left(\varepsilon^{N}\right), & \alpha\left(t_{0}\right)=\alpha_{0},
\end{array}\right.
$$

Let $f_{\mathrm{gy}}: \Omega_{\mathrm{gy}} \rightarrow \mathbb{R}_{+}$denote the unique function which is constant along the solutions of $\left(P^{\varepsilon}\right)$, with initial condition $f_{\mathrm{gy}}\left(t=t_{0}\right)=f_{0, \mathrm{gy}}$ strictly positive. Since $\left(P^{\varepsilon}\right)$ is merely a reformulation of the initial-value problem (9) in the coordinates $\widehat{q}_{\text {gy }}$ via the map $\tau_{\text {gy }}^{\varepsilon}$, we have $f_{\mathrm{gy}}=f \circ \tau_{\mathrm{gy}}^{\varepsilon}$, where $f$ is the unique solution of the Vlasov equation (1) with initial condition $f_{0}=f_{0, \mathrm{gy}} \circ\left(\tau_{\mathrm{gy}}^{\varepsilon}\right)^{-1}$. Regarding existence of $f_{\mathrm{gy}}$ we remark:

Lemma 2. A solution of the problem $\left(P^{\varepsilon}\right)$ exists, is unique and continuous on the interval $\left[t_{0}, t_{0}+T\right]$, where $T$ is given in Lemma 1 .

Proof. This follows from Lemma 1 and the fact that $\left(P^{\varepsilon}\right)$ is equivalent to the initial value problem (9) if $\widehat{q}_{\mathrm{gy}, 0}=\left(\tau_{\mathrm{gy}}^{\varepsilon}\right)^{-1}\left(x_{0}, v_{0}, t_{0}\right)$. The inverse of the algebraic GY-map $\tau_{\mathrm{gy}}^{\varepsilon}=\tau^{\prime} \circ \tau^{\varepsilon} \circ \widehat{\tau}$ exists at least locally due to the fact $\tau^{\varepsilon}$ is continuously differentiable because all generators are (Theorem 1).

If one truncates the residual terms of order $O\left(\varepsilon^{k \geq N}\right)$ in (49), one obtains the "decoupled dynamics", which are the Euler-Lagrange equations of the truncated Lagrangian (48). A 
decoupled solution is denoted with an overbar, hence the decoupled problem is written as

$$
\left(\bar{P}^{\varepsilon}\right)\left\{\begin{array}{lll}
\frac{\mathrm{d} \bar{r}}{\mathrm{~d} t}=\frac{1}{B_{\|}^{*}}\left(\bar{q}_{\|}+\varepsilon \frac{\partial \delta H^{(N)}}{\partial q_{\|}}\right) B^{*}+\varepsilon \frac{1}{B_{\|}^{*}} E^{*} \times b_{0}, & \bar{r}\left(t_{0}\right)=\bar{r}_{0}, \\
\frac{\mathrm{d} \bar{q}_{\|}}{\mathrm{d} t}=\frac{1}{B_{\|}^{*}} B^{*} \cdot E^{*}, & \bar{q}_{\|}\left(t_{0}\right)=\bar{q}_{\| 0} \\
\frac{\mathrm{d} \bar{\mu}}{\mathrm{d} t}=0, & \overline{\widehat{\mu}}\left(t_{0}\right)=\overline{\widehat{\mu}}_{0}, \\
\frac{\mathrm{d} \bar{\alpha}}{\mathrm{d} t}=\frac{\left|B_{0}\right|}{\varepsilon}+\frac{\partial \delta H^{(N)}}{\partial \widehat{\mu}}, & \bar{\alpha}\left(t_{0}\right)=\bar{\alpha}_{0},
\end{array}\right.
$$

Definition 5. (Gyrokinetic equation.) A solution $F: \widehat{\Omega}_{\mathrm{gy}} \rightarrow \mathbb{R}_{+}$of a gyrokinetic equation is a strictly positive function, constant along the solutions of the decoupled dynamics (50),

$$
\left\{\begin{array}{l}
\frac{\partial F}{\partial t}+\frac{\mathrm{d} \bar{r}}{\mathrm{~d} t} \cdot \frac{\partial F}{\partial r}+\frac{\mathrm{d} \bar{q}_{\|}}{\mathrm{d} t} \frac{\partial F}{\partial q_{\|}}+\frac{\mathrm{d} \bar{\alpha}}{\mathrm{d} t} \frac{\partial F}{\partial \alpha}=0 \\
F\left(t=t_{0}\right)=F_{0}
\end{array}\right.
$$

Lemma 3. The gyro-average $\langle F\rangle$ and fluctuations $\widetilde{F}$ of a solution to (51) evolve independently in time (decoupling); they satisfy

$$
\begin{array}{ll}
\frac{\partial\langle F\rangle}{\partial t}+\frac{\mathrm{d} \bar{r}}{\mathrm{~d} t} \cdot \frac{\partial\langle F\rangle}{\partial r}+\frac{\mathrm{d} \bar{q}_{\|}}{\mathrm{d} t} \frac{\partial\langle F\rangle}{\partial q_{\|}}=0, & \langle F\rangle\left(t=t_{0}\right)=\left\langle F_{0}\right\rangle, \\
\frac{\partial \widetilde{F}}{\partial t}+\frac{\mathrm{d} \bar{r}}{\mathrm{~d} t} \cdot \frac{\partial \widetilde{F}}{\partial r}+\frac{\mathrm{d} \bar{q}_{\|}}{\mathrm{d} t} \frac{\partial \widetilde{F}}{\partial q_{\|}}+\frac{\mathrm{d} \bar{\alpha}}{\mathrm{d} t} \frac{\partial \widetilde{F}}{\partial \alpha}=0, & \widetilde{F}\left(t=t_{0}\right)=\widetilde{F}_{0} .
\end{array}
$$

Proof. This is an immediate consequence of the definition 4 of the gyro-average and the fact that the direction field (right-hand-side) in (50) is independent of the gyro-angle $\bar{\alpha}$.

In what follows we denote by $\mathbf{z}:=\left(r, q_{\|}, \widehat{\mu}\right)$ the slow components of the phase space variables $\widehat{q}_{\mathrm{gy}}$. In the decoupled dynamics the "slow trajectories" $\overline{\mathbf{z}}(t):=\left(\bar{r}(t), \bar{q}_{\|}(t), \overline{\widehat{\mu}}(t)\right)$ evolve independently from the gyro-angle $\bar{\alpha}(t)$, which varies with a frequency $1 / \varepsilon$. From (49) and (50) we can extract two subproblems for the slow variables,

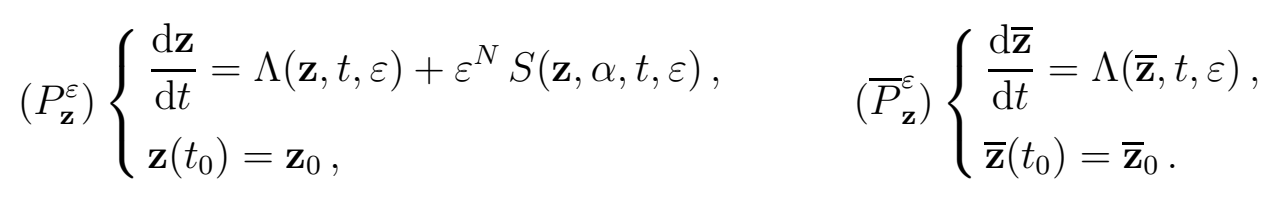

Here, $\Lambda$ stands for the direction field for the slow variables in (50) and $S$ comprises the residual terms $O\left(\varepsilon^{k \geq N}\right)$ of (49) for the slow variables. 
Remark 3. The direction field $\Lambda$ is independent of $\alpha$. From assumption 1 we deduce that it is a $C^{1}$-function of $(\mathbf{z}, t, \varepsilon)$. In particular, $\Lambda$ is Lipschitz in $\mathbf{z}$, uniformly in $(t, \varepsilon)$,

$$
\|\Lambda(\mathbf{y}, t, \varepsilon)-\Lambda(\mathbf{z}, t, \varepsilon)\| \leq \ell_{\Lambda}\|\mathbf{y}-\mathbf{z}\|,
$$

for some vector norm $\|\cdot\|$, where the Lipschitz constant $\ell_{\Lambda}$ is independent of $(t, \varepsilon)$.

Remark 4. The residual term $S$ depends on $\alpha$. It is obtained from the $O\left(\varepsilon^{N+1}\right)$-terms in the Lagrangian (47) via the Euler-Lagrange equations. Since these $O\left(\varepsilon^{N+1}\right)$-terms are the residuals in the Taylor expansion (62), they are $C^{1}$-functions of $(\mathbf{z}, \alpha, t)$. Therefore $S$, being a power series in $\varepsilon$, is continuous in $(\mathbf{z}, \alpha, t, \varepsilon)$.

Lemma 4. Consider the problems $\left(P_{\mathbf{z}}^{\varepsilon}\right)$ and $\left(\bar{P}_{\mathbf{z}}^{\varepsilon}\right)$ for the slow $G Y$-variables on the interval $t \in I=\left[t_{0}, t_{1}\right]$ with $t_{1} \leq T$ (Lemma 2), then

$$
\|\overline{\mathbf{z}}(t)-\mathbf{z}(t)\| \leq \varepsilon^{N} \frac{\|S\|_{\infty, \varepsilon}}{\ell_{\Lambda}}\left(e^{\ell_{\Lambda}\left(t-t_{0}\right)}-1\right)+\left\|\overline{\mathbf{z}}_{0}-\mathbf{z}_{0}\right\| e^{\ell_{\Lambda}\left(t-t_{0}\right)}
$$

where $\|S\|_{\infty, \varepsilon}=\max _{\widehat{\Omega}_{\mathrm{gy}} \times\left(0, \varepsilon_{\max }\right]}\|S\|=O(1)$ as $\varepsilon \rightarrow 0$.

Proof. The proof is written in section 5.3.

Theorem 2. Let $\langle F\rangle$ denote the unique solution of the averaged part (52) of the gyrokinetic equation on the interval $I=\left[t_{0}, t_{1}\right]$, with initial condition $\left\langle F_{0}\right\rangle$. Moreover, let $f_{\mathrm{gy}}=f \circ \tau_{\mathrm{gy}}^{\varepsilon}$, where $f$ is the unique solution of the Vlasov equation (1) with initial data $f_{0}$, hence $f_{0, \mathrm{gy}}=f_{0} \circ \tau_{\mathrm{gy}}^{\varepsilon}$, where $\tau_{\mathrm{gy}}^{\varepsilon}$ denotes the transformation (46) of order $N$. Suppose

1. $\left\langle F_{0}\right\rangle=\left\langle f_{0, \mathrm{gy}}\right\rangle, \quad$ Lipschitz with constant $\ell_{0}$,

2. $f_{0, \mathrm{gy}}=\left\langle f_{0, \mathrm{gy}}\right\rangle+\varepsilon^{N} \widetilde{f}_{0, \mathrm{gy}}$, with $\widetilde{f}_{0, \mathrm{gy}}=O(1)$ as $\varepsilon \rightarrow 0$ continuous .

Then, denoting $\mathbf{z}=\left(r, q_{\|}, \widehat{\mu}\right)$, for $t \in I$ one has

$$
\max _{\mathbf{z}, \alpha}\left|\langle F\rangle(\mathbf{z}, t)-f_{\mathrm{gy}}(\mathbf{z}, \alpha, t)\right| \leq \varepsilon^{N} C(t),
$$

with

$$
C(t)=\ell_{0} \frac{\|S\|_{\infty, \varepsilon}}{\ell_{\Lambda}}\left(e^{\ell_{\Lambda}\left(t-t_{0}\right)}-1\right)+\max _{\mathbf{z}, \alpha}\left|\tilde{f}_{0, \mathrm{gy}}(\mathbf{z}, \alpha)\right|,
$$

where the function $S$ is the one from Lemma 4.

Proof. Let $\Phi_{s, t}: \widehat{\Omega}_{\mathrm{gy}} \rightarrow \widehat{\Omega}_{\mathrm{gy}}$ stand for the local flow map ${ }^{1}$ of problem $\left(P^{\varepsilon}\right)$, i.e $\Phi_{s, t}(\mathbf{z}, \alpha)$ is the solution of $\left(P^{\varepsilon}\right)$ at time $s$ which is at $(\mathbf{z}, \alpha)$ at time $t$, and $\Phi_{t, t}=\operatorname{id}_{\widehat{\Omega}_{\mathrm{gy}}}$. We shall denote the "slow" components of the flow by $\mathbf{Z}_{s, t}$, corresponding to $\mathbf{z}$ for the slow variables, i.e.

\footnotetext{
${ }^{1}$ We have $\Phi_{t, s}=\Phi_{s, t}^{-1}$, since $\operatorname{id}_{\widehat{\Omega}_{\mathrm{gy}}}=\Phi_{t, t}=\Phi_{t, s} \circ \Phi_{s, t}$ by the semi-group property of the local flow.
} 
$\mathbf{Z}_{t, t}(\mathbf{z}, \alpha)=\mathbf{z}$ and $\mathbf{Z}_{t, t_{0}}\left(\mathbf{z}_{0}, \alpha_{0}\right)=\mathbf{z}(t)$, solution of the subproblem $\left(P_{\mathbf{z}}^{\varepsilon}\right)$ written in $(54)$. Using that $f_{\mathrm{gy}}$ is constant along solutions of $\left(P^{\varepsilon}\right)$ we may write

$$
\begin{aligned}
f_{\mathrm{gy}}(\mathbf{z}, \alpha, t) & =f_{\mathrm{gy}}\left(\Phi_{t, t}(\mathbf{z}, \alpha), t\right) \\
& =f_{\mathrm{gy}}\left(\Phi_{t_{0}, t}(\mathbf{z}, \alpha), t_{0}\right) \\
& =f_{0, \mathrm{gy}}\left(\Phi_{t_{0}, t}(\mathbf{z}, \alpha)\right) \\
& =\left\langle f_{0, \mathrm{gy}}\right\rangle\left(\mathbf{Z}_{t_{0}, t}(\mathbf{z}, \alpha)\right)+\varepsilon^{N} \widetilde{f}_{0, \mathrm{gy}}\left(\Phi_{t_{0}, t}(\mathbf{z}, \alpha)\right) .
\end{aligned}
$$

Our aim is to compare this expression to $\langle F\rangle$, solution of (52). For this let us denote by $\overline{\mathbf{Z}}_{s, t}$ the flow map of the subproblem $\left(\bar{P}_{\mathbf{z}}^{\varepsilon}\right)$ from $(54)$, i.e. $\overline{\mathbf{Z}}_{t, t}(\overline{\mathbf{z}})=\overline{\mathbf{z}}$ and $\overline{\mathbf{Z}}_{t, t_{0}}\left(\overline{\mathbf{z}}_{0}\right)=\overline{\mathbf{z}}(t)$. Since $\langle F\rangle$ is constant along solutions of $\left(\bar{P}_{\mathbf{z}}^{\varepsilon}\right)$ we have

$$
\begin{aligned}
\langle F\rangle(\mathbf{z}, t) & =\langle F\rangle\left(\overline{\mathbf{Z}}_{t, t}(\mathbf{z}), t\right) \\
& =\langle F\rangle\left(\overline{\mathbf{Z}}_{t_{0}, t}(\mathbf{z}), t_{0}\right) \\
& =\left\langle F_{0}\right\rangle\left(\overline{\mathbf{Z}}_{t_{0}, t}(\mathbf{z})\right) \\
& =\left\langle f_{0, \mathrm{gy}}\right\rangle\left(\overline{\mathbf{Z}}_{t_{0}, t}(\mathbf{z})\right) .
\end{aligned}
$$

Therefore, using the Lipschitz continuity of $\left\langle f_{0, g y}\right\rangle$ we obtain

$$
\begin{aligned}
\left|\langle F\rangle(\mathbf{z}, t)-f_{\mathrm{gy}}(\mathbf{z}, \alpha, t)\right| & =\left|\left\langle f_{0, \mathrm{gy}}\right\rangle\left(\overline{\mathbf{Z}}_{t_{0}, t}(\mathbf{z})\right)-\left\langle f_{0, \mathrm{gy}}\right\rangle\left(\mathbf{Z}_{t_{0}, t}(\mathbf{z}, \alpha)\right)-\varepsilon^{N} \tilde{f}_{0, \mathrm{gy}}\left(\Phi_{t_{0}, t}(\mathbf{z}, \alpha)\right)\right| \\
& \leq\left|\left\langle f_{0, \mathrm{gy}}\right\rangle\left(\overline{\mathbf{Z}}_{t_{0}, t}(\mathbf{z})\right)-\left\langle f_{0, \mathrm{gy}}\right\rangle\left(\mathbf{Z}_{t_{0}, t}(\mathbf{z}, \alpha)\right)\right|+\varepsilon^{N}\left|\widetilde{f}_{0, \mathrm{gy}}\left(\Phi_{t_{0}, t}(\mathbf{z}, \alpha)\right)\right| \\
& \left.\leq \ell_{0}|| \overline{\mathbf{Z}}_{t_{0}, t}(\mathbf{z})\right)-\mathbf{Z}_{t_{0}, t}(\mathbf{z}, \alpha)||+\varepsilon^{N}\left|\widetilde{f}_{0, \mathrm{gy}}\left(\Phi_{t_{0}, t}(\mathbf{z}, \alpha)\right)\right| .
\end{aligned}
$$

The continuity of $\widetilde{f}_{0, \text { gy }}$ leads to a bound for the second term. The difference in the flow functions can be estimated from Lemma 4,

$$
\left.\forall(\mathbf{z}, \alpha, t) \in \Omega_{\mathrm{gy}} \cap I: \quad \| \overline{\mathbf{Z}}_{t_{0}, t}(\mathbf{z})\right)-\mathbf{Z}_{t_{0}, t}(\mathbf{z}, \alpha) \| \leq \varepsilon^{N} \frac{\|S\|_{\infty, \varepsilon}}{\ell_{\Lambda}}\left(e^{\ell_{\Lambda}\left(t-t_{0}\right)}-1\right) .
$$

\section{Expressions for $\delta \mu^{(N)}, \delta H^{(N)}$ and the generators}

Theorem 1 states the existence of algebraic GY-maps $\tau^{\varepsilon}$ that lead to the reduced dynamics implied by the Lagrangian (44). Here we give some concrete examples of such transformations for the two different scalings (8) of $\varepsilon_{B}$ and for the orders $N=1$ and $N=2$, respectively. We stress that the choice for the generators in these transformations is not unique for two reasons: 
1. the equivalence of Lagrangians that differ by a "total time derivative" allows us to add or subtract such a term,

2. gyro-averages of the generators could be kept in the Lagrangian rather than in the transformation; one then still obtains a set of decoupled equations of motion, albeit a rather different one, c.f. the discussion in remark 5 .

Moreover, we state the derived expressions for the correction $\delta \mu^{(N)}$ to the magnetic moment, the map $\widehat{\tau}$ between $\widehat{\mu}$ and $q_{\perp}$, as well as the correction $\delta H^{(N)}$ to the Hamiltonian. The proof of the following Lemmas is left as an exercise to the reader; it consists of performing the steps described in the proof of Theorem 1.

Lemma 5. (Small background variations $\varepsilon_{B}=\varepsilon, N=1$.) In this case possible generators read

$$
\begin{aligned}
\varrho_{1} & =\frac{q_{\perp}}{\left|B_{0}\right|} a_{0}, \\
G_{1}^{\|} & =-\frac{q_{\perp}}{\left|B_{0}\right|} B_{1} \cdot c_{0}, \\
G_{1}^{\perp} & =\frac{q_{\|}}{\left|B_{0}\right|} B_{1} \cdot c_{0}+\frac{1}{\left|B_{0}\right|} E \cdot a_{0}, \\
\varrho_{2} & =\left[\frac{q_{\|}}{\left|B_{0}\right|^{2}} B_{1} \cdot c_{0}-\frac{q_{\perp}}{\left|B_{0}\right|^{2}} B_{1} \cdot b_{0}+\frac{1}{\left|B_{0}\right|^{2}} E \cdot a_{0}\right] a_{0}+\left(\varrho_{2} \cdot b_{0}\right) b_{0}+\frac{q_{\perp}}{\left|B_{0}\right|} G_{1}^{\theta} c_{0},
\end{aligned}
$$

where $\varrho_{2} \cdot b_{0}$ and $G_{1}^{\theta}$ are arbitrary. This leads to $\delta \mu^{(1)}=0$, therefore $\widehat{\mu}=\mu$, the map $\widehat{\tau}: \widehat{\mu} \mapsto q_{\perp}$ is given by $q_{\perp}=\sqrt{2 \widehat{\mu}\left|B_{0}\right|}$ and the Hamiltonian correction is zero,

$$
\delta H^{(1)}=0 .
$$

Lemma 6. (Small background variations $\varepsilon_{B}=\varepsilon, N=2$.) In this case possible generators are the functions in (55) along with

$$
\begin{aligned}
\varrho_{2} \cdot b_{0}= & \frac{\partial S_{2}^{* *}}{\partial q_{\|}}, \\
G_{1}^{\theta}= & \frac{\left|B_{0}\right|}{q_{\perp}} \frac{\partial S_{2}^{* *}}{\partial q_{\perp}}, \\
G_{2}^{\|}= & \left(\varrho_{2} \times B_{1}\right) \cdot b_{0}-\mathcal{Q}_{2}^{* *} \cdot b_{0}, \\
G_{2}^{\perp}= & -\frac{q_{\|}}{q_{\perp}} G_{2}^{\|}+\frac{1}{q_{\perp}} \varrho_{2} \cdot E+\frac{q_{\perp}}{2\left|B_{0}\right|^{2}} a_{0} \cdot \nabla E \cdot a_{0}+\frac{1}{q_{\perp}} \frac{\partial S_{2}^{* *}}{\partial t}, \\
\varrho_{3}= & \frac{1}{\left|B_{0}\right|}\left[G_{2}^{\perp}-\left(\varrho_{2} \times B_{1}\right) \cdot c_{0}+\mathcal{Q}_{2}^{* *} \cdot c_{0}\right] a_{0} \\
& +\left(\varrho_{3} \cdot b_{0}\right) b_{0}+\frac{1}{\left|B_{0}\right|}\left[\left(\varrho_{2} \times B_{1}\right) \cdot a_{0}-\mathcal{Q}_{2}^{* *} \cdot a_{0}\right] c_{0},
\end{aligned}
$$


where $\mathcal{Q}_{2}^{* *}$ is given by

$$
\begin{aligned}
\mathcal{Q}_{2}^{* *}= & \frac{q_{\perp}^{2}}{2\left|B_{0}\right|^{2}}\left(a_{0} \cdot \nabla B_{0}\right) \times a_{0}+\frac{q_{\perp}^{2}}{2\left|B_{0}\right|^{2}}\left(a_{0} \cdot \nabla B_{1}\right) \times a_{0} \\
& -\frac{q_{\|} q_{\perp}}{\left|B_{0}\right|} a_{0} \times\left(\nabla \times b_{0}\right)-\frac{q_{\perp}^{2}}{\left|B_{0}\right|} a_{0} \times\left(\nabla \times c_{0}\right)-G_{1}^{\perp} G_{1}^{\theta} a_{0}-q_{\perp} G_{2}^{\theta} a_{0} \\
& -\frac{q_{\perp}}{2}\left(G_{1}^{\theta}\right)^{2} c_{0}-\frac{q_{\perp}^{2}}{2\left|B_{0}\right|} \mathrm{R} .
\end{aligned}
$$

with $\mathrm{R}=\nabla a_{0} \cdot c_{0}=\nabla e_{2} \cdot e_{1}$ denoting the gyro-gauge term, $\varrho_{3} \cdot b_{0}$ and $G_{2}^{\theta}$ are arbitrary and $S_{2}^{* *}$ reads

$$
S_{2}^{* *}=-\frac{q_{\|} q_{\perp}}{\left|B_{0}\right|^{2}} B_{1} \cdot a_{0}+\frac{q_{\perp}}{\left|B_{0}\right|^{2}} E \cdot c_{0}
$$

This leads to

$$
\delta \mu^{(2)}=\frac{q_{\perp}^{2}}{2\left|B_{0}\right|} \underbrace{\frac{\left(-B_{1} \cdot b_{0}\right)}{\left|B_{0}\right|}}_{=: \sigma^{* *}}
$$

where $\sigma^{* *}$ is independent of $q_{\perp}$. Therefore, $\widehat{\mu}=\mu\left(1+\varepsilon \sigma^{* *}\right)$, the map $\widehat{\tau}: \widehat{\mu} \mapsto q_{\perp}$ is given by $q_{\perp}=\sqrt{2 \widehat{\mu}\left|B_{0}\right| /\left(1+\varepsilon \sigma^{* *}\right)}$ and the Hamiltonian correction reads

$$
\delta H^{(2)}=-\widehat{\mu}\left|B_{0}\right| \frac{\sigma^{* *}}{1+\varepsilon \sigma^{* *}} .
$$

Remark 5. Standard second-order gyrokinetic Lagrangians in the long-wavelength approximation [44], used for example in the codes GENE and ORB5 [7,20], can be recovered from Lemma 6. However, our choice of the generators differs from the conventional ones and leads to simpler equations of motion. For example, the polarization term $|\nabla \phi|^{2}$ usually appearing in gyrokinetic Hamiltonian functions at second order has been included in the generator $G_{2}^{\perp}$ in our formalism; it is hidden in the term $\varrho_{2} \cdot E$ and does not play a role in the particle dynamics, which are derived from the Lagrangian (44) and are thus simpler. The polarization term re-appears only through the use of the GY-transformation, respectively its inverse, in the transformation to $x, v$-phase-space. This reflects our general strategy of keeping the particle dynamics as simple as possible by keeping a maximum number of terms in the generating functions, instead of the Lagrangian (see the proof section for more details). A new class of gyrokinetic numerical schemes based on this strategy could be envisioned. 
Lemma 7. $\left(\varepsilon_{B}=1, N=1\right.$.) In this case possible generators read

$$
\begin{aligned}
\varrho_{1}= & \frac{q_{\perp}}{\left|B_{0}\right|} a_{0} \\
G_{1}^{\|}= & -\frac{q_{\perp}}{\left|B_{0}\right|} B_{1} \cdot c_{0}+\frac{q_{\perp}^{2}}{2\left|B_{0}\right|} a_{0} \cdot \nabla b_{0} \cdot c_{0}-\frac{q_{\|} q_{\perp}}{\left|B_{0}\right|}\left(\nabla \times b_{0}\right) \cdot c_{0}-\frac{q_{\perp}^{2}}{2\left|B_{0}\right|} \mathrm{R} \cdot b_{0} \\
G_{1}^{\perp}= & -\frac{q_{\|}}{q_{\perp}} G_{1}^{\|}+\frac{1}{\left|B_{0}\right|} a_{0} \cdot E \\
\varrho_{2}= & {\left[\frac{G_{1}^{\perp}}{\left|B_{0}\right|}-\frac{q_{\perp}}{\left|B_{0}\right|^{2}} B_{1} \cdot b_{0}-\frac{q_{\perp}^{2}}{2\left|B_{0}\right|^{3}} a_{0} \cdot \nabla\left|B_{0}\right|-\frac{q_{\|} q_{\perp}}{\left|B_{0}\right|^{2}}\left(\nabla \times b_{0}\right) \cdot b_{0}+\frac{q_{\perp}^{2}}{2\left|B_{0}\right|^{2}} \mathrm{R} \cdot c_{0}\right] a_{0} } \\
& +\left(\varrho_{2} \cdot b_{0}\right) b_{0}+\left[\frac{q_{\perp} G_{1}^{\theta}}{\left|B_{0}\right|}+\frac{q_{\perp}^{2}}{2\left|B_{0}\right|^{2}} \mathrm{R} \cdot a_{0}\right] c_{0}
\end{aligned}
$$

where $\mathrm{R}=\nabla a_{0} \cdot c_{0}=\nabla e_{2} \cdot e_{1}$ is the gyro-gauge term and $\varrho_{2} \cdot b_{0}$ and $G_{1}^{\theta}$ are arbitrary. This leads to $\delta \mu^{(1)}=0$, therefore $\widehat{\mu}=\mu$, the map $\widehat{\tau}: \widehat{\mu} \mapsto q_{\perp}$ is given by $q_{\perp}=\sqrt{2 \widehat{\mu}\left|B_{0}\right|}$ and the Hamiltonian correction is zero,

$$
\delta H^{(1)}=0
$$

Lemma 8. $\left(\varepsilon_{B}=1, N=2\right.$.) In this case possible generators are the functions in (58) along with

$$
\begin{aligned}
\varrho_{2} \cdot b_{0}= & \frac{\partial S_{2}^{*}}{\partial q_{\|}}-\frac{q_{\perp}^{2}}{2\left|B_{0}\right|^{2}} a_{0} \cdot \nabla b_{0} \cdot a_{0}, \\
G_{1}^{\theta}= & \frac{\left|B_{0}\right|}{q_{\perp}} \frac{\partial S_{2}^{*}}{\partial q_{\perp}}+\frac{q_{\perp}}{2\left|B_{0}\right|} a_{0} \cdot \mathrm{R}, \\
G_{2}^{\|}= & \left(\varrho_{2} \times B_{1}\right) \cdot b_{0}-\mathcal{Q}_{2}^{*} \cdot b_{0}, \\
G_{2}^{\perp}= & -\frac{q_{\|}}{q_{\perp}} G_{2}^{\|}+\frac{1}{q_{\perp}} \varrho_{2} \cdot E+\frac{q_{\perp}}{2\left|B_{0}\right|^{2}} a_{0} \cdot \nabla E \cdot a_{0}+\frac{1}{q_{\perp}} \frac{\partial S_{2}^{*}}{\partial t}, \\
\varrho_{3}= & \frac{1}{\left|B_{0}\right|}\left[G_{2}^{\perp}-\left(\varrho_{2} \times B_{1}\right) \cdot c_{0}+\mathcal{Q}_{2}^{*} \cdot c_{0}\right] a_{0} \\
& +\left(\varrho_{3} \cdot b_{0}\right) b_{0}+\frac{1}{\left|B_{0}\right|}\left[\left(\varrho_{2} \times B_{1}\right) \cdot a_{0}-\mathcal{Q}_{2}^{*} \cdot a_{0}\right] c_{0},
\end{aligned}
$$

where $\varrho_{3} \cdot b_{0}$ and $G_{2}^{\theta}$ are arbitrary and $S_{2}^{*}$ reads

$$
\begin{aligned}
S_{2}^{*}= & -\frac{q_{\|} q_{\perp}}{\left|B_{0}\right|^{2}} B_{1} \cdot a_{0}+\frac{q_{\perp}}{\left|B_{0}\right|^{2}} E \cdot c_{0}+\frac{q_{\|} q_{\perp}^{2}}{4\left|B_{0}\right|^{2}}\left(a_{0} \cdot \nabla b_{0} \cdot a_{0}\right) \\
& -\frac{q_{\|}^{2} w_{\perp}}{\left|B_{0}\right|^{2}}\left(\nabla \times b_{0}\right) \cdot a_{0}-\frac{q_{\perp}^{3}}{3\left|B_{0}\right|^{3}} c_{0} \cdot \nabla\left|B_{0}\right|+\frac{q_{\perp}^{3}}{2\left|B_{0}\right|^{2}}\left(\nabla \times a_{0}\right) \cdot b_{0} .
\end{aligned}
$$


This leads to

$$
\delta \mu^{(2)}=\frac{q_{\perp}^{2}}{2\left|B_{0}\right|} \underbrace{\left[-\frac{1}{\left|B_{0}\right|}\left(B_{1} \cdot b_{0}+\frac{1}{2} q_{\|}\left(\nabla \times b_{0}\right) \cdot b_{0}\right)+\frac{2 q_{\|}}{\left|B_{0}\right|} \mathrm{R} \cdot b_{0}\right]}_{=: \sigma^{*}},
$$

where $\sigma^{*}$ is independent of $q_{\perp}$. Therefore, $\widehat{\mu}=\mu\left(1+\varepsilon \sigma^{*}\right)$, the map $\widehat{\tau}: \widehat{\mu} \mapsto q_{\perp}$ is given by $q_{\perp}=\sqrt{2 \widehat{\mu}\left|B_{0}\right| /\left(1+\varepsilon \sigma^{*}\right)}$ and the Hamiltonian correction reads

$$
\delta H^{(2)}=-\widehat{\mu}\left|B_{0}\right| \frac{\sigma^{*}}{1+\varepsilon \sigma^{*}} .
$$

The term $\mathcal{Q}_{2}^{*}$ is given by

$$
\begin{aligned}
\mathcal{Q}_{2}^{*}= & \frac{q_{\perp}}{2\left|B_{0}\right|}\left(\varrho_{2} \cdot \nabla B_{0}\right) \times a_{0}+\frac{q_{\perp}}{2\left|B_{0}\right|}\left(a_{0} \cdot \nabla B_{0}\right) \times \varrho_{2}+\frac{q_{\perp}^{2}}{2\left|B_{0}\right|^{2}}\left(a_{0} \cdot \nabla B_{1}\right) \times a_{0} \\
& -G_{1}^{\|} \frac{q_{\perp}}{\left|B_{0}\right|} a_{0} \times\left(\nabla \times b_{0}\right)-G_{1}^{\perp} \frac{q_{\perp}}{\left|B_{0}\right|} a_{0} \times\left(\nabla \times c_{0}\right)-G_{1}^{\perp} G_{1}^{\theta} a_{0} \\
& -q_{\|} \varrho_{2} \times\left(\nabla \times b_{0}\right)-q_{\perp} \varrho_{2} \times\left(\nabla \times c_{0}\right)-q_{\perp} G_{2}^{\theta} a_{0} \\
& -G_{1}^{\theta} \frac{q_{\perp}^{2}}{\left|B_{0}\right|} a_{0} \cdot \nabla a_{0}-\frac{q_{\perp}}{2}\left(G_{1}^{\theta}\right)^{2} c_{0}+\frac{q_{\perp}^{2}}{\left|B_{0}\right|^{2}} G_{1}^{\theta} \nabla\left|B_{0}\right|-\frac{q_{\perp}^{2}}{6\left|B_{0}\right|^{2}} a_{0} \times\left(a_{0} \cdot \nabla\right)^{2} B_{0} \\
& -\frac{q_{\|}}{2} \varrho_{1} \times\left(\nabla^{\prime} \times\left(\varrho_{1} \cdot \nabla b_{0}\right)\right)-\frac{q_{\perp}}{2} \varrho_{1} \times\left(\nabla^{\prime} \times\left(\varrho_{1} \cdot \nabla c_{0}\right)\right) \\
& +\frac{1}{2} \nabla B_{0} \cdot\left(\varrho_{2} \times \varrho_{1}\right)-\frac{q_{\perp}}{\left|B_{0}\right|} \nabla\left|B_{0}\right|-q_{\perp} \nabla a_{0} \cdot\left(\varrho_{2} \times b_{0}\right) \\
& -\frac{q_{\perp}^{3}}{3\left|B_{0}\right|^{3}}\left(\nabla a_{0} \times a_{0}\right) \cdot\left(a_{0} \cdot \nabla B_{0}\right)-\frac{q_{\perp}^{2}}{2\left|B_{0}\right|^{2}}\left(\nabla a_{0} \times a_{0}\right) \cdot B_{1} \\
& -\frac{q_{\|} q_{\perp}^{2}}{2\left|B_{0}\right|^{2}}\left(\nabla a_{0} \times a_{0}\right) \cdot\left(\nabla \times b_{0}\right)-\frac{q_{\perp}^{3}}{2\left|B_{0}\right|^{2}}\left(\nabla a_{0} \times a_{0}\right) \cdot\left(\nabla \times c_{0}\right)+\frac{\partial S_{2}^{*}}{\partial r}
\end{aligned}
$$

where $\nabla^{\prime}$ only acts on $\nabla b_{0}$ and $\nabla c_{0}$.

\section{Proofs}

\subsection{Proof of Proposition 1}

The proof is split into three sections, with the following startegy in mind: first, for $\left(q_{\mathrm{gy}}, \dot{q}_{\mathrm{gy}}\right)$ fixed, we consider the Lagrangian $L^{\varepsilon}$ in (40) as a function of $\varepsilon$ and apply Taylor's theorem to write a series expansion in $\varepsilon$ and estimate the remainder. In part two we compute the coefficients of this series expansion. This step involves a lot of algebra. Finally, we rewrite the series expansion of the Lagrangian so as to arrive at a gauge-invariant representation. 
Let us introduce the following notation for the components of the GT (38)-(39),

$$
\begin{array}{rlrl}
x_{\varepsilon}\left(q_{\mathrm{gy}}\right) & :=\sum_{n=0}^{N+1} \varepsilon^{n} \varrho_{n}\left(q_{\mathrm{gy}}\right), & \varrho_{0}=r, \\
v_{\|, \varepsilon}\left(q_{\mathrm{gy}}\right) & :=\sum_{n=0}^{N+1} \varepsilon^{n} G_{n}^{\|}\left(q_{\mathrm{gy}}\right), & G_{0}^{\|}=q_{\|}, \\
v_{\perp, \varepsilon}\left(q_{\mathrm{gy}}\right):=\sum_{n=0}^{N+1} \varepsilon^{n} G_{n}^{\perp}\left(q_{\mathrm{gy}}\right), & G_{0}^{\perp}=q_{\perp}, \\
\theta_{\varepsilon}\left(q_{\mathrm{gy}}\right):=\sum_{n=0}^{N+1} \varepsilon^{n} G_{n}^{\theta}\left(q_{\mathrm{gy}}\right), & G_{0}^{\theta}=\alpha, \\
\dot{x}_{\varepsilon}\left(q_{\mathrm{gy}}, \dot{q}_{\mathrm{gy}}\right) & :=\sum_{n=0}^{N+1} \varepsilon^{n} \dot{\varrho}_{n}\left(q_{\mathrm{gy}}, \dot{q}_{\mathrm{gy}}\right), & & \dot{\varrho}_{0}=\dot{r},
\end{array}
$$

where the time coordinate $t$ rests untransformed. For $\left(q_{\text {gy }}, \dot{q}_{\text {gy }}\right)$ fixed, we consider the Lagrangian (40) as a function of $\varepsilon$, split into three parts, $L^{\varepsilon}=\varphi_{0} / \varepsilon+\varphi_{1}-\varphi_{2} \dot{t}$, with

$$
\begin{aligned}
\varphi_{0}(\varepsilon) & :=\dot{x}_{\varepsilon} \cdot A_{0}\left(x_{\varepsilon}\right) . \\
\varphi_{1}(\varepsilon) & :=v_{\|, \varepsilon} \dot{x}_{\varepsilon} \cdot b_{0}\left(x_{\varepsilon}\right)+v_{\perp, \varepsilon} \dot{x}_{\varepsilon} \cdot c_{0}\left(x_{\varepsilon}, \theta_{\varepsilon}\right)+\dot{x}_{\varepsilon} \cdot A_{1}\left(t, x_{\varepsilon}\right), \\
\varphi_{2}(\varepsilon) & :=\frac{v_{\|, \varepsilon}^{2}}{2}+\frac{v_{\perp, \varepsilon}^{2}}{2}+\phi\left(t, x_{\varepsilon}\right) .
\end{aligned}
$$

From assumption 1 we have $A_{0} \in C^{N+3}\left(\Omega_{x}\right)$ and thus $\varphi_{0} \in C^{N+3}\left(\left[0, \varepsilon_{\max }\right]\right)$, since $\dot{x}_{\varepsilon}$ is a polynomial in $\varepsilon$. Also, $b_{0}, c_{0}, A_{1}, \phi \in C^{N+2}\left(\Omega_{x}\right)$ which implies $\varphi_{1}, \varphi_{2} \in C^{N+2}\left(\left[0, \varepsilon_{\max }\right]\right)$. Hence, we may apply Taylor's theorem and write

$$
\begin{aligned}
& \varphi_{0}(\varepsilon)=\sum_{j=0}^{N+1} \frac{\varepsilon^{j}}{j !} \varphi_{0}^{(j)}(0)+O\left(\varepsilon^{N+2}\right), \\
& \varphi_{1}(\varepsilon)=\sum_{j=0}^{N} \frac{\varepsilon^{j}}{j !} \varphi_{1}^{(j)}(0)+O\left(\varepsilon^{N+1}\right), \\
& \varphi_{2}(\varepsilon)=\sum_{j=0}^{N} \frac{\varepsilon^{j}}{j !} \varphi_{2}^{(j)}(0)+O\left(\varepsilon^{N+1}\right),
\end{aligned}
$$

where $\varphi^{(j)}$ denotes the $j$-th derivative of $\varphi$ with respect to $\varepsilon$. The remainders are still $C^{1}$. This kind of regularity is necessary in the proof of Lemma 4 where, in order to apply the Gronwall's lemma 11, one needs the residual terms in the Euler-Lagrange equations to be continuous, which is guaranteed by the remainders being in $C^{1}$. 


\subsubsection{Taylor coefficients}

Let us now compute the coefficients of the $\varepsilon$-polynomials (62). For $j=0$ one has

$$
\begin{aligned}
& \varphi_{0}(0)=A_{0}(r) \cdot \dot{r}, \\
& \varphi_{1}(0)=\left[q_{\|} b_{0}(r)+q_{\perp} c_{0}(r)+A_{1}(t, r)\right] \cdot \dot{r}, \\
& \varphi_{2}(0)=\frac{q_{\|}^{2}}{2}+\frac{q_{\perp}^{2}}{2}+\phi(t, r) .
\end{aligned}
$$

To compute the derivatives of order $j$, we use the Leibniz rule,

$$
(\varphi \chi)^{(j)}=\sum_{l=0}^{j}\left(\begin{array}{l}
j \\
l
\end{array}\right) \varphi^{(j-l)} \chi^{(l)} .
$$

For polynomials in $\varepsilon$ we use the formula

$$
\left[\sum_{n=0}^{N+1} \varepsilon^{n} \varrho_{n}\right]^{(j)}=\sum_{n=j}^{N+1} \frac{n !}{(n-j) !} \varepsilon^{n-j} \varrho_{n},
$$

which leads to

$$
\left[\sum_{n=0}^{N+1} \varepsilon^{n} \varrho_{n}\right]^{(j)}(0)=j ! \varrho_{j} .
$$

We shall use the nable symbol to denote the gradient with respect to the position variable, $\nabla \equiv \partial / \partial x$. In order to write the Taylor expansion of a function $b_{0}(r+s(\varepsilon, r))$ around $r$ we define the operator $\nabla^{\prime}$, which acts only on the function $b_{0}$ and not on $s$. Hence,

$$
\begin{aligned}
b_{0}(r+s(\varepsilon, r)) & =b_{0}(r)+\sum_{i} s_{i} \frac{\partial b_{0}(r)}{\partial x_{i}}+\frac{1}{2} \sum_{i, j} s_{i} s_{j} \frac{\partial^{2} b_{0}(r)}{\partial x_{i} \partial x_{j}}+\frac{1}{6} \sum_{i, j, k} s_{i} s_{j} s_{k} \frac{\partial^{3} b_{0}(r)}{\partial x_{i} \partial x_{j} \partial x_{k}}+\ldots \\
& =b_{0}(r)+s \cdot \nabla^{\prime} b_{0}(r)+\frac{1}{2}\left(s \cdot \nabla^{\prime}\right)^{2} b_{0}(r)+\frac{1}{6}\left(s \cdot \nabla^{\prime}\right)^{3} b_{0}(r)+\ldots
\end{aligned}
$$

With the Leibniz rule (64), for $j \geq 1$ one computes

$$
\begin{aligned}
b_{0}^{(j)}\left(x_{\varepsilon}\right) & =\left(x_{\varepsilon}^{(1)} \cdot \nabla^{\prime} b_{0}\right)^{(j-1)} \\
& =\sum_{k_{1}=0}^{j-1}\left(\begin{array}{c}
j-1 \\
k_{1}
\end{array}\right)\left(x_{\varepsilon}^{\left(j-k_{1}\right)} \cdot \nabla^{\prime}\right) b_{0}^{\left(k_{1}\right)} \\
& =x_{\varepsilon}^{(j)} \cdot \nabla^{\prime} b_{0}+\sum_{k_{1}=1}^{j-1}\left(\begin{array}{c}
j-1 \\
k_{1}
\end{array}\right)\left(x_{\varepsilon}^{\left(j-k_{1}\right)} \cdot \nabla^{\prime}\right) b_{0}^{\left(k_{1}\right)} .
\end{aligned}
$$


Here and in the following, for sums we will use the convention

$$
\sum_{k=k_{\mathrm{start}}}^{j} \ldots=0 \quad \text { if } \quad j<k_{\mathrm{start}} .
$$

Using the rule (67) two times yields

$$
\begin{aligned}
b_{0}^{(j)}\left(x_{\varepsilon}\right)= & x_{\varepsilon}^{(j)} \cdot \nabla^{\prime} b_{0}+\sum_{k_{1}=1}^{j-1}\left(\begin{array}{c}
j-1 \\
k_{1}
\end{array}\right)\left(x_{\varepsilon}^{\left(j-k_{1}\right)} \cdot \nabla^{\prime}\right)\left(x_{\varepsilon}^{\left(k_{1}\right)} \cdot \nabla^{\prime}\right) b_{0} \\
& +\sum_{k_{1}=2}^{j-1}\left(\begin{array}{c}
j-1 \\
k_{1}
\end{array}\right)\left(x_{\varepsilon}^{\left(j-k_{1}\right)} \cdot \nabla^{\prime}\right) \sum_{k_{2}=1}^{k_{1}-1}\left(\begin{array}{c}
k_{1}-1 \\
k_{2}
\end{array}\right)\left(x_{\varepsilon}^{\left(k_{1}-k_{2}\right)} \cdot \nabla^{\prime}\right) b_{0}^{\left(k_{2}\right)} .
\end{aligned}
$$

We note that $x_{\varepsilon}^{(k)}$ is a function of $\varepsilon$ and $q_{\mathrm{gy}}$, and that $\nabla^{\prime}$ only acts on $b_{0}$. Applying the rule (67) a third time leads to

$$
\begin{aligned}
b_{0}^{(j)}\left(x_{\varepsilon}\right)= & x_{\varepsilon}^{(j)} \cdot \nabla^{\prime} b_{0}+\sum_{k_{1}=1}^{j-1}\left(\begin{array}{c}
j-1 \\
k_{1}
\end{array}\right)\left(x_{\varepsilon}^{\left(j-k_{1}\right)} \cdot \nabla^{\prime}\right)\left(x_{\varepsilon}^{\left(k_{1}\right)} \cdot \nabla^{\prime}\right) b_{0} \\
& +\sum_{k_{1}=2}^{j-1}\left(\begin{array}{c}
j-1 \\
k_{1}
\end{array}\right)\left(x_{\varepsilon}^{\left(j-k_{1}\right)} \cdot \nabla^{\prime}\right) \sum_{k_{2}=1}^{k_{1}-1}\left(\begin{array}{c}
k_{1}-1 \\
k_{2}
\end{array}\right)\left(x_{\varepsilon}^{\left(k_{1}-k_{2}\right)} \cdot \nabla^{\prime}\right)\left(x_{\varepsilon}^{\left(k_{2}\right)} \cdot \nabla^{\prime}\right) b_{0} \\
& +\sum_{k_{1}=3}^{j-1}\left(\begin{array}{c}
j-1 \\
k_{1}
\end{array}\right)\left(x_{\varepsilon}^{\left(j-k_{1}\right)} \cdot \nabla^{\prime}\right) \sum_{k_{2}=2}^{k_{1}-1}\left(\begin{array}{c}
k_{1}-1 \\
k_{2}
\end{array}\right)\left(x_{\varepsilon}^{\left(k_{1}-k_{2}\right)} \cdot \nabla^{\prime}\right) \sum_{k_{3}=1}^{k_{2}-1}\left(\begin{array}{c}
k_{2}-1 \\
k_{3}
\end{array}\right) \times \\
& \times\left(x_{\varepsilon}^{\left(k_{2}-k_{3}\right)} \cdot \nabla^{\prime}\right) b_{0}^{\left(k_{3}\right)} .
\end{aligned}
$$

At each iteration, the starting index of sums in the last term gets raised by one due to the sum convention (68). One can thus apply the rule (67) $j-1$ times, until the last term becomes

$$
\begin{aligned}
& \sum_{k_{1}=j-1}^{j-1} \sum_{k_{2}=j-2}^{j-2} \ldots \sum_{k_{j-1}=1}^{1}\left(\begin{array}{c}
j-1 \\
k_{1}
\end{array}\right)\left(\begin{array}{c}
k_{1}-1 \\
k_{2}
\end{array}\right) \ldots\left(\begin{array}{c}
1 \\
k_{j-1}
\end{array}\right) \\
& \underbrace{\left(x_{\varepsilon}^{\left(j-k_{1}\right)} \cdot \nabla^{\prime}\right)\left(x_{\varepsilon}^{\left(k_{1}-k_{2}\right)} \cdot \nabla^{\prime}\right) \ldots\left(x_{\varepsilon}^{\left(k_{j-2}-k_{j-1}\right)} \cdot \nabla^{\prime}\right)}_{j-1 \text { times }}\left(x_{\varepsilon}^{\left(k_{j-1}\right)} \cdot \nabla^{\prime}\right) b_{0} \\
& =\left(x_{\varepsilon}^{(1)} \cdot \nabla^{\prime}\right)^{j} b_{0} .
\end{aligned}
$$

Therefore, by applying the rule (67) recursively, for $j \geq 1$ we can write the result in the compact form

$$
b_{0}^{(j)}\left(x_{\varepsilon}\right)=x_{\varepsilon}^{(j)} \cdot \nabla b_{0}+\mathcal{R}_{j-1}^{\varepsilon},
$$


with $\mathcal{R}_{0}^{\varepsilon}=0$ and, for $j \geq 2$, with $\mathcal{R}_{j-1}^{\varepsilon}$ defined by

$$
\begin{aligned}
\mathcal{R}_{j-1}^{\varepsilon} & :=\sum_{k_{1}=1}^{j-1}\left(\begin{array}{c}
j-1 \\
k_{1}
\end{array}\right)\left(x_{\varepsilon}^{\left(j-k_{1}\right)} \cdot \nabla^{\prime}\right)\left(x_{\varepsilon}^{\left(k_{1}\right)} \cdot \nabla^{\prime}\right) b_{0} \\
& +\sum_{k_{1}=2}^{j-1} \sum_{k_{2}=1}^{k_{1}-1}\left(\begin{array}{c}
j-1 \\
k_{1}
\end{array}\right)\left(\begin{array}{c}
k_{1}-1 \\
k_{2}
\end{array}\right)\left(x_{\varepsilon}^{\left(j-k_{1}\right)} \cdot \nabla^{\prime}\right)\left(x_{\varepsilon}^{\left(k_{1}-k_{2}\right)} \cdot \nabla^{\prime}\right)\left(x_{\varepsilon}^{\left(k_{2}\right)} \cdot \nabla^{\prime}\right) b_{0} \\
& +\ldots \\
& +\sum_{k_{1}=j-2 \geq 3}^{j-1} \sum_{k_{2}=j-3}^{k_{1}-1} \ldots \sum_{k_{j-2}=1}^{k_{j-3}-1}\left(\begin{array}{c}
j-1 \\
k_{1}
\end{array}\right)\left(\begin{array}{c}
k_{1}-1 \\
k_{2}
\end{array}\right) \ldots\left(\begin{array}{c}
k_{j-3}-1 \\
k_{j-2}
\end{array}\right) \\
& \quad\left(x_{\varepsilon}^{\left(j-k_{1}\right)} \cdot \nabla^{\prime}\right)\left(x_{\varepsilon}^{\left(k_{1}-k_{2}\right)} \cdot \nabla^{\prime}\right) \ldots\left(x_{\varepsilon}^{\left(k_{j-3}-k_{j-2}\right)} \cdot \nabla^{\prime}\right)\left(x_{\varepsilon}^{\left(k_{j-2}\right)} \cdot \nabla^{\prime}\right) b_{0} \\
& +\left(x_{\varepsilon}^{(1)} \cdot \nabla^{\prime}\right)^{j} b_{0} .
\end{aligned}
$$

Since we need the derivatives (69) evaluated at $\varepsilon=0$, using (66) leads to

$$
\left.b_{0}^{(j)}\left(x_{\varepsilon}\right)\right|_{\varepsilon=0}=j !\left[\varrho_{j} \cdot \nabla b_{0}(r)+\mathcal{R}_{j-1}\left(b_{0}\right)\right], \quad j \geq 1,
$$

with $\mathcal{R}_{0}=0$ and, for $j \geq 2$, with the term $\mathcal{R}_{j-1}$ defined by

$$
\begin{aligned}
\mathcal{R}_{j-1}\left(b_{0}\right) & :=\frac{1}{j !} \mathcal{T}_{j-1}^{0}\left(b_{0}\right)=\sum_{k_{1}=1}^{j-1} \frac{\left(j-k_{1}\right)}{j}\left(\varrho_{j-k_{1}} \cdot \nabla^{\prime}\right)\left(\varrho_{k_{1}} \cdot \nabla^{\prime}\right) b_{0}(r) \\
& +\sum_{k_{1}=2}^{j-1} \sum_{k_{2}=1}^{k_{1}-1} \frac{\left(j-k_{1}\right)\left(k_{1}-k_{2}\right)}{j k_{1}}\left(\varrho_{j-k_{1}} \cdot \nabla^{\prime}\right)\left(\varrho_{k_{1}-k_{2}} \cdot \nabla^{\prime}\right)\left(\varrho_{k_{2}} \cdot \nabla^{\prime}\right) b_{0}(r) \\
& +\ldots \\
& +\sum_{k_{1}=j-2 \geq 3}^{j-1} \sum_{k_{2}=j-3}^{k_{1}-1} \ldots \sum_{k_{j-2}=1}^{k_{j-3}-1} \frac{\left(j-k_{1}\right)\left(k_{1}-k_{2}\right) \ldots\left(k_{j-3}-k_{j-2}\right)}{j k_{1} k_{2} \ldots k_{j-3}} \\
& +\frac{1}{j !}\left(\varrho_{1} \cdot \nabla^{\prime}\right)^{j} b_{0}(r) .
\end{aligned}
$$


The computation of $c_{0}^{(j)}\left(x_{\varepsilon}, \theta_{\varepsilon}\right)$ is done in a similar way, i.e. we start as in (67),

$$
\begin{aligned}
c_{0}^{(j)}\left(x_{\varepsilon}, \theta_{\varepsilon}\right) & =\left(x_{\varepsilon}^{(1)} \cdot \nabla^{\prime} c_{0}+\theta_{\varepsilon}^{(1)} \frac{\partial}{\partial \theta} c_{0}\right)^{(j-1)} \\
& =\sum_{k_{1}=0}^{j-1}\left(\begin{array}{c}
j-1 \\
k_{1}
\end{array}\right)\left(x_{\varepsilon}^{\left(j-k_{1}\right)} \cdot \nabla^{\prime}+\theta_{\varepsilon}^{\left(j-k_{1}\right)} \frac{\partial}{\partial \theta}\right) c_{0}^{\left(k_{1}\right)} \\
& =x_{\varepsilon}^{(j)} \cdot \nabla^{\prime} c_{0}+\theta_{\varepsilon}^{(j)} \frac{\partial}{\partial \theta} c_{0}+\sum_{k_{1}=1}^{j-1}\left(\begin{array}{c}
j-1 \\
k_{1}
\end{array}\right)\left(x_{\varepsilon}^{\left(j-k_{1}\right)} \cdot \nabla^{\prime}+\theta_{\varepsilon}^{\left(j-k_{1}\right)} \frac{\partial}{\partial \theta}\right) c_{0}^{\left(k_{1}\right)},
\end{aligned}
$$

Applying this rule twice yields

$$
\begin{aligned}
c_{0}^{(j)}=x_{\varepsilon}^{(j)} \cdot \nabla^{\prime} c_{0}+\theta_{\varepsilon}^{(j)} \frac{\partial}{\partial \theta} c_{0}+\sum_{k_{1}=1}^{j-1}\left(\begin{array}{c}
j-1 \\
k_{1}
\end{array}\right) & \left(x_{\varepsilon}^{\left(j-k_{1}\right)} \cdot \nabla^{\prime}+\theta_{\varepsilon}^{\left(j-k_{1}\right)} \frac{\partial}{\partial \theta}\right) \\
& \times\left(x_{\varepsilon}^{\left(k_{1}\right)} \cdot \nabla^{\prime} c_{0}+\theta_{\varepsilon}^{\left(k_{1}\right)} \frac{\partial}{\partial \theta} c_{0}\right) \\
+ & \sum_{k_{1}=2}^{j-1}\left(\begin{array}{c}
j-1 \\
k_{1}
\end{array}\right)\left(x_{\varepsilon}^{\left(j-k_{1}\right)} \cdot \nabla^{\prime}+\theta_{\varepsilon}^{\left(j-k_{1}\right)} \frac{\partial}{\partial \theta}\right) \sum_{k_{2}=1}^{k_{1}-1}\left(\begin{array}{c}
k_{1}-1 \\
k_{2}
\end{array}\right)\left(x_{\varepsilon}^{\left(k_{1}-k_{2}\right)} \cdot \nabla^{\prime}+\theta_{\varepsilon}^{\left(k_{1}-k_{2}\right)} \frac{\partial}{\partial \theta}\right) c^{\left(k_{2}\right)} .
\end{aligned}
$$

We remind the reader again that $x_{\varepsilon}^{(k)}$ as well as $\theta_{\varepsilon}^{(k)}$ are functions of $\varepsilon$ and $q_{\mathrm{gy}}$ and that $\nabla^{\prime}$ and $\partial / \partial \theta$ only act on $c_{0}$. We can apply the rule (73) $j-1$ times, until the last term becomes

$$
\begin{aligned}
& \sum_{k_{1}=j-1}^{j-1} \sum_{k_{2}=j-2}^{j-2} \ldots \sum_{k_{j-1}=1}^{1}\left(\begin{array}{c}
j-1 \\
k_{1}
\end{array}\right)\left(\begin{array}{c}
k_{1}-1 \\
k_{2}
\end{array}\right) \ldots\left(\begin{array}{c}
1 \\
k_{j-1}
\end{array}\right) \\
& \quad \times\left(x_{\varepsilon}^{\left(j-k_{1}\right)} \cdot \nabla^{\prime}+\theta_{\varepsilon}^{\left(j-k_{1}\right)} \frac{\partial}{\partial \theta}\right)\left(x_{\varepsilon}^{\left(k_{1}-k_{2}\right)} \cdot \nabla^{\prime}+\theta_{\varepsilon}^{\left(k_{1}-k_{2}\right)} \frac{\partial}{\partial \theta}\right) \ldots \\
& \quad \times\left(x_{\varepsilon}^{\left(k_{j-2}-k_{j-1}\right)} \cdot \nabla^{\prime}+\theta_{\varepsilon}^{\left(k_{j-2}-k_{j-1}\right)} \frac{\partial}{\partial \theta}\right)\left(x_{\varepsilon}^{\left(k_{j-1}\right)} \cdot \nabla^{\prime}+\theta_{\varepsilon}^{\left(k_{j-1}\right)} \frac{\partial}{\partial \theta}\right) c_{0} \\
& =\left(x_{\varepsilon}^{(1)} \cdot \nabla^{\prime}+\theta_{\varepsilon}^{(1)} \frac{\partial}{\partial \theta}\right)^{j} c_{0} .
\end{aligned}
$$

Hence, with $\partial c_{0} / \partial \alpha=-a_{0}$, in analogy to (71) we arrive at

$$
\left.c_{0}^{(j)}\left(x_{\varepsilon}, \theta_{\varepsilon}\right)\right|_{\varepsilon=0}=j !\left[\varrho_{j} \cdot \nabla c_{0}(r, \theta)-G_{j}^{\theta} a_{0}(r, \theta)+\mathcal{R}_{j-1}^{\alpha}\left(c_{0}\right)\right], \quad j \geq 1,
$$


with $\mathcal{R}_{0}^{\alpha}=0$ and, for $j \geq 2$, with the term $\mathcal{R}_{j-1}^{\alpha}$ defined by

$$
\begin{aligned}
& \mathcal{R}_{j-1}^{\alpha}\left(c_{0}\right):=\sum_{k_{1}=1}^{j-1} \frac{\left(j-k_{1}\right)}{j}\left(\varrho_{j-k_{1}} \cdot \nabla^{\prime}+G_{j-k_{1}}^{\theta} \frac{\partial}{\partial \theta}\right)\left(\varrho_{k_{1}} \cdot \nabla^{\prime}+G_{k_{1}}^{\theta} \frac{\partial}{\partial \theta}\right) c_{0} \\
& +\sum_{k_{1}=2}^{j-1} \sum_{k_{2}=1}^{k_{1}-1} \frac{\left(j-k_{1}\right)\left(k_{1}-k_{2}\right)}{j k_{1}}\left(\varrho_{j-k_{1}} \cdot \nabla^{\prime}+G_{j-k_{1}}^{\theta} \frac{\partial}{\partial \theta}\right)\left(\varrho_{k_{1}-k_{2}} \cdot \nabla^{\prime}+G_{k_{1}-k_{2}}^{\theta} \frac{\partial}{\partial \theta}\right) \\
& \times\left(\varrho_{k_{2}} \cdot \nabla^{\prime}+G_{k_{2}}^{\theta} \frac{\partial}{\partial \theta}\right) c_{0} \\
& +\ldots \\
& +\sum_{k_{1}=j-2 \geq 3}^{j-1} \sum_{k_{2}=j-3}^{k_{1}-1} \ldots \sum_{k_{j-2}=1}^{k_{j-3}-1} \frac{\left(j-k_{1}\right)\left(k_{1}-k_{2}\right) \ldots\left(k_{j-3}-k_{j-2}\right)}{j k_{1} k_{2} \ldots k_{j-3}} \\
& \times\left(\varrho_{j-k_{1}} \cdot \nabla^{\prime}+G_{j-k_{1}}^{\theta} \frac{\partial}{\partial \theta}\right)\left(\varrho_{k_{1}-k_{2}} \cdot \nabla^{\prime}+G_{k_{1}-k_{2}}^{\theta} \frac{\partial}{\partial \theta}\right) \ldots\left(\varrho_{k_{j-2}} \cdot \nabla^{\prime}+G_{k_{j-2}}^{\theta} \frac{\partial}{\partial \theta}\right) c_{0} \\
& +\frac{1}{j !}\left(\varrho_{1} \cdot \nabla^{\prime}+G_{1}^{\theta} \frac{\partial}{\partial \theta}\right)^{j} c_{0}
\end{aligned}
$$

where derivatives of $c_{0}$ are avaluated at $(r, \alpha)$.

We have now all the material to compute the $j$-h derivative at $\varepsilon=0$ of $\varphi_{0}, \varphi_{1}$ and $\varphi_{1}$, defined in (61), which are the coefficients of the Taylor expansions (62). Applying the Leibniz rule (64) twice, for the first term in (61b) we obtain

$$
\begin{aligned}
{\left[v_{\|, \varepsilon} \dot{x}_{\varepsilon} \cdot b_{0}\left(x_{\varepsilon}\right)\right]^{(j)}(0) } & =\left\{\sum_{l=0}^{j}\left(\begin{array}{l}
j \\
l
\end{array}\right)\left[v_{\|, \varepsilon} \dot{x}_{\varepsilon}\right]^{(j-l)} \cdot b_{0}^{(l)}\left(x_{\varepsilon}\right)\right\}_{\varepsilon=0} \\
& =\left\{\sum_{l=0}^{j}\left(\begin{array}{l}
j \\
l
\end{array}\right)\left[\sum_{m=0}^{j-l}\left(\begin{array}{c}
j-l \\
m
\end{array}\right) v_{\|, \varepsilon}^{(j-l-m)} \dot{x}_{\varepsilon}^{(m)}\right] \cdot b_{0}^{(l)}\left(x_{\varepsilon}\right)\right\}_{\varepsilon=0} .
\end{aligned}
$$

Since $b_{0}^{(l)}\left(x_{\varepsilon}\right)$ at $\varepsilon=0$ is given by the formula (71) for $l \geq 1$, we single out the summand with $l=0$, and insert (66) to obtain, for $j \geq 1$,

$$
\begin{aligned}
& {\left[v_{\|, \varepsilon} \dot{x}_{\varepsilon} \cdot b_{0}\left(x_{\varepsilon}\right)\right]^{(j)}(0)=\sum_{m=0}^{j} \frac{j !}{m !(j-m) !}(j-m) ! G_{j-m}^{\|} m ! \dot{\varrho}_{m} \cdot b_{0}} \\
& +\sum_{l=1}^{j} \frac{j !}{l !(j-l) !}\left[\sum_{m=0}^{j-l} \frac{(j-l) !}{m !(j-l-m) !}(j-l-m) ! G_{j-l-m}^{\|} m ! \dot{\varrho}_{m}\right] \cdot l !\left[\varrho_{l} \cdot \nabla b_{0}+\mathcal{R}_{l-1}\left(b_{0}\right)\right] \\
& =j ! \sum_{m=0}^{j} G_{j-m}^{\|} \dot{\varrho}_{m} \cdot b_{0}+j ! \sum_{l=1}^{j}\left[\sum_{m=0}^{j-l} G_{j-l-m}^{\|} \dot{\varrho}_{m}\right] \cdot\left[\varrho_{l} \cdot \nabla b_{0}+\mathcal{R}_{l-1}\left(b_{0}\right)\right],
\end{aligned}
$$


With the same reasoning, using the result (74), for the second term in (61b) one obtains

$$
\begin{aligned}
& {\left[v_{\perp, \varepsilon} \dot{x}_{\varepsilon} \cdot c_{0}\left(x_{\varepsilon}, \theta_{\varepsilon}\right)\right]^{(j)}(0)} \\
& \quad=j ! \sum_{m=0}^{j} G_{j-m}^{\perp} \dot{\varrho}_{m} \cdot c_{0}+j ! \sum_{l=1}^{j}\left[\sum_{m=0}^{j-l} G_{j-l-m}^{\perp} \dot{\varrho}_{m}\right] \cdot\left[\varrho_{l} \cdot \nabla c_{0}-G_{l}^{\theta} a_{0}+\mathcal{R}_{l-1}^{\alpha}\left(c_{0}\right)\right],
\end{aligned}
$$

For the third term in (61b) we apply the Leibniz rule (64) once to compute

$$
\begin{aligned}
{\left[\dot{x}_{\varepsilon} \cdot A_{1}\left(t, x_{\varepsilon}\right)\right]^{(j)}(0) } & =\left\{\sum_{l=0}^{j}\left(\begin{array}{l}
j \\
l
\end{array}\right) \dot{x}_{\varepsilon}^{(j-l)} \cdot A_{1}^{(l)}\left(t, x_{\varepsilon}\right)\right\}_{\varepsilon=0} \\
& =j ! \dot{\varrho}_{j} \cdot A_{1}+j ! \sum_{l=1}^{j} \dot{\varrho}_{j-l} \cdot\left[\varrho_{l} \cdot \nabla A_{1}+\mathcal{R}_{l-1}\left(A_{1}\right)\right] .
\end{aligned}
$$

From this result we can also compute the derivatives of $\varphi_{0}$ given in (61a),

$$
\frac{\varphi_{0}^{(j)}(0)}{j !}=\dot{\varrho}_{j} \cdot A_{0}+\sum_{l=1}^{j} \dot{\varrho}_{j-l} \cdot\left[\varrho_{l} \cdot \nabla A_{0}+\mathcal{R}_{l-1}\left(A_{0}\right)\right]
$$

For $\varphi_{1}$ given in (61b), using also the previous result (76), we finally obtain

$$
\begin{aligned}
\frac{\varphi_{1}^{(j)}(0)}{j !} & =\sum_{m=0}^{j} G_{j-m}^{\|} \dot{\varrho}_{m} \cdot b_{0}+\sum_{l=1}^{j}\left[\sum_{m=0}^{j-l} G_{j-l-m}^{\|} \dot{\varrho}_{m}\right] \cdot\left[\varrho_{l} \cdot \nabla b_{0}+\mathcal{R}_{l-1}\left(b_{0}\right)\right] \\
& +\sum_{m=0}^{j} G_{j-m}^{\perp} \dot{\varrho}_{m} \cdot c_{0}+\sum_{l=1}^{j}\left[\sum_{m=0}^{j-l} G_{j-l-m}^{\perp} \dot{\varrho}_{m}\right] \cdot\left[\varrho_{l} \cdot \nabla c_{0}-G_{l}^{\theta} a_{0}+\mathcal{R}_{l-1}^{\alpha}\left(c_{0}\right)\right] \\
& +\dot{\varrho}_{j} \cdot A_{1}+\sum_{l=1}^{j} \dot{\varrho}_{j-l} \cdot\left[\varrho_{l} \cdot \nabla A_{1}+\mathcal{R}_{l-1}\left(A_{1}\right)\right]
\end{aligned}
$$

For $\varphi_{2}$ given in (61c), using (66) and (71), one obtains

$$
\frac{\varphi_{2}^{(j)}(0)}{j !}=q_{\|} G_{j}^{\|}+q_{\perp} G_{j}^{\perp}+\varrho_{j} \cdot \nabla \phi(r)+\mathcal{R}_{j-1}(\phi)
$$

We shall transform the expressions (79)-(80) a bit further. In particular, in the sum over $l$ in (79) and in the last line of (80) we single out the term with $l=j$. Additionally, in the sums over $m$ in (80) we single out the terms with $m=0$. For $j \geq 1$, this leads to

$$
\frac{\varphi_{0}^{(j)}(0)}{j !}=\dot{\varrho}_{j} \cdot A_{0}+\dot{r} \cdot\left[\varrho_{j} \cdot \nabla A_{0}+\mathcal{R}_{j-1}\left(A_{0}\right)\right]+\sum_{l=1}^{j-1} \dot{\varrho}_{j-l} \cdot\left[\varrho_{l} \cdot \nabla A_{0}+\mathcal{R}_{l-1}\left(A_{0}\right)\right]
$$


and to

$$
\begin{aligned}
& \frac{\varphi_{1}^{(j)}(0)}{j !}=G_{j}^{\|} \dot{r} \cdot b_{0}+\sum_{m=1}^{j} G_{j-m}^{\|} \dot{\varrho}_{m} \cdot b_{0}+\sum_{l=1}^{j} G_{j-l}^{\|}\left[\varrho_{l} \cdot \nabla b_{0}+\mathcal{R}_{l-1}\left(b_{0}\right)\right] \cdot \dot{r} \\
& +\sum_{l=1}^{j-1}\left[\sum_{m=1}^{j-l} G_{j-l-m}^{\|} \dot{\varrho}_{m}\right] \cdot\left[\varrho_{l} \cdot \nabla b_{0}+\mathcal{R}_{l-1}\left(b_{0}\right)\right] \\
& +G_{j}^{\perp} \dot{r} \cdot c_{0}+\sum_{m=1}^{j} G_{j-m}^{\perp} \dot{\varrho}_{m} \cdot c_{0}+\sum_{l=1}^{j} G_{j-l}^{\perp}\left[\varrho_{l} \cdot \nabla c_{0}-G_{l}^{\theta} a_{0}+\mathcal{R}_{l-1}^{\alpha}\left(c_{0}\right)\right] \cdot \dot{r} \\
& +\sum_{l=1}^{j-1}\left[\sum_{m=1}^{j-l} G_{j-l-m}^{\perp} \dot{\varrho}_{m}\right] \cdot\left[\varrho_{l} \cdot \nabla c_{0}-G_{l}^{\theta} a_{0}+\mathcal{R}_{l-1}^{\alpha}\left(c_{0}\right)\right] \\
& +\dot{\varrho}_{j} \cdot A_{1}+\dot{r} \cdot\left[\varrho_{j} \cdot \nabla A_{1}+\mathcal{R}_{j-1}\left(A_{1}\right)\right]+\sum_{l=1}^{j-1} \dot{\varrho}_{j-l} \cdot\left[\varrho_{l} \cdot \nabla A_{1}+\mathcal{R}_{l-1}\left(A_{1}\right)\right] .
\end{aligned}
$$

It will be convenient to eliminate the terms $\dot{\varrho}_{m}$ from the first and the third line of (83), using the equivalence of Lagrangians from definition (3):

$$
\begin{aligned}
& \sum_{m=1}^{j} G_{j-m}^{\|} \dot{\varrho}_{m} \cdot b_{0}+\sum_{l=1}^{j} G_{j-l}^{\|} \varrho_{l} \cdot \nabla b_{0} \cdot \dot{r} \\
& =\sum_{m=1}^{j}\left[\frac{\mathrm{d}}{\mathrm{d} s}\left(G_{j-m}^{\|} \varrho_{m} \cdot b_{0}\right)-\dot{G}_{j-m}^{\|}\left(\varrho_{m} \cdot b_{0}\right)-G_{j-m}^{\|} \dot{r} \cdot\left(\varrho_{m} \times\left(\nabla \times b_{0}\right)\right)\right],
\end{aligned}
$$

and, respectively,

$$
\begin{aligned}
& \sum_{m=1}^{j} G_{j-m}^{\perp} \dot{\varrho}_{m} \cdot c_{0}+\sum_{l=1}^{j} G_{j-l}^{\perp}\left(\varrho_{l} \cdot \nabla c_{0}-G_{l}^{\theta} a_{0}\right) \cdot \dot{r} \\
& =\sum_{m=1}^{j} G_{j-m}^{\perp}\left[\dot{\varrho}_{m} \cdot c_{0}+\left(\varrho_{m} \cdot \nabla c_{0}-G_{m}^{\theta} a_{0}\right) \cdot \dot{r}\right] \\
& =\sum_{m=1}^{j} G_{j-m}^{\perp}\left[\frac{\mathrm{d}}{\mathrm{d} s}\left(\varrho_{m} \cdot c_{0}\right)-\left(\dot{r} \cdot \nabla c_{0}-\dot{\alpha} a_{0}\right) \cdot \varrho_{m}+\left(\varrho_{m} \cdot \nabla c_{0}-G_{m}^{\theta} a_{0}\right) \cdot \dot{r}\right] \\
& =\sum_{m=1}^{j}\left[\frac{\mathrm{d}}{\mathrm{d} s}\left(G_{j-m}^{\perp} \varrho_{m} \cdot c_{0}\right)-\dot{G}_{j-m}^{\perp}\left(\varrho_{m} \cdot c_{0}\right)-G_{j-m}^{\perp} \dot{r} \cdot\left(\varrho_{m} \times\left(\nabla \times c_{0}\right)\right)\right. \\
& \left.\quad+G_{j-m}^{\perp} \dot{\alpha}\left(\varrho_{m} \cdot a_{0}\right)-G_{j-m}^{\perp} G_{m}^{\theta} a_{0} \cdot \dot{r}\right],
\end{aligned}
$$


which leads to

$$
\begin{aligned}
& \frac{\varphi_{1}^{(j)}(0)}{j !} \sim G_{j}^{\|} \dot{r} \cdot b_{0}+G_{j}^{\perp} \dot{r} \cdot c_{0}-\sum_{m=1}^{j}\left[\dot{G}_{j-m}^{\|}\left(\varrho_{m} \cdot b_{0}\right)+G_{j-m}^{\|} \dot{r} \cdot\left(\varrho_{m} \times\left(\nabla \times b_{0}\right)\right)\right] \\
& +\sum_{l=1}^{j-1}\left[\sum_{m=1}^{j-l} G_{j-l-m}^{\|} \dot{\varrho}_{m}\right] \cdot\left[\varrho_{l} \cdot \nabla b_{0}+\mathcal{R}_{l-1}\left(b_{0}\right)\right]+\sum_{l=2}^{j} G_{j-l}^{\|} \mathcal{R}_{l-1}\left(b_{0}\right) \cdot \dot{r} \\
& -\sum_{m=1}^{j}\left[\dot{G}_{j-m}^{\perp}\left(\varrho_{m} \cdot c_{0}\right)+G_{j-m}^{\perp} \dot{r} \cdot\left(\varrho_{m} \times\left(\nabla \times c_{0}\right)\right)-G_{j-m}^{\perp} \dot{\alpha}\left(\varrho_{m} \cdot a_{0}\right)+G_{j-m}^{\perp} G_{m}^{\theta} a_{0} \cdot \dot{r}\right] \\
& +\sum_{l=1}^{j-1}\left[\sum_{m=1}^{j-l} G_{j-l-m}^{\perp} \dot{\varrho}_{m}\right] \cdot\left[\varrho_{l} \cdot \nabla c_{0}-G_{l}^{\theta} a_{0}+\mathcal{R}_{l-1}^{\alpha}\left(c_{0}\right)\right]+\sum_{l=2}^{j} G_{j-l}^{\perp} \mathcal{R}_{l-1}^{\alpha}\left(c_{0}\right) \cdot \dot{r} \\
& +\dot{\varrho}_{j} \cdot A_{1}+\dot{r} \cdot\left[\varrho_{j} \cdot \nabla A_{1}+\mathcal{R}_{j-1}\left(A_{1}\right)\right]+\sum_{l=1}^{j-1} \dot{\varrho}_{j-l} \cdot\left[\varrho_{l} \cdot \nabla A_{1}+\mathcal{R}_{l-1}\left(A_{1}\right)\right] .
\end{aligned}
$$

The Lagrangian $L^{\varepsilon}$ now reads

$$
\begin{aligned}
L^{\varepsilon} & =\frac{\varphi_{0}(\varepsilon)}{\varepsilon}+\varphi_{1}(\varepsilon)-\varphi_{2}(\varepsilon) \dot{t} \\
& =\sum_{j=0}^{N+1} \frac{\varepsilon^{j-1}}{j !} \varphi_{0}^{(j)}(0)+\sum_{j=0}^{N} \frac{\varepsilon^{j}}{j !} \varphi_{1}^{(j)}(0)-\sum_{j=0}^{N} \frac{\varepsilon^{j}}{j !} \varphi_{2}^{(j)}(0) \dot{t}+O\left(\varepsilon^{N+1}\right) \\
& =\frac{\varphi_{0}(0)}{\varepsilon}+\varphi_{0}^{(1)}(0)+\varphi_{1}(0)-\varphi_{2}(0) \dot{t}+\sum_{j=1}^{N} \varepsilon^{j}\left[\frac{\varphi_{0}^{(j+1)}(0)}{(j+1) !}+\frac{\varphi_{1}^{(j)}(0)}{(j) !}-\frac{\varphi_{2}^{(j)}(0)}{(j) !} \dot{t}\right]+O\left(\varepsilon^{N+1}\right),
\end{aligned}
$$

where we used (62) to estimate the remainder. The terms $\varphi_{0}(0), \varphi_{1}(0)$ and $\varphi_{2}(0)$ are given in (63). From (82) one computes

$$
\varphi_{0}^{(1)}(0)=\dot{\varrho}_{1} \cdot A_{0}+\varrho_{1} \cdot \nabla A_{0} \cdot \dot{r} .
$$

For $j \geq 1$, from (81),(82) and (86) one computes

$$
\begin{aligned}
& \frac{\varphi_{0}^{(j+1)}(0)}{(j+1) !}+\frac{\varphi_{1}^{(j)}(0)}{(j) !}-\frac{\varphi_{2}^{(j)}(0)}{(j) !} \dot{t}=\dot{\varrho}_{j+1} \cdot A_{0}+\varrho_{j+1} \cdot \nabla A_{0} \cdot \dot{r}+\dot{\varrho}_{j} \cdot A_{1}+\varrho_{j} \cdot \nabla A_{1} \cdot \dot{r} \\
& +\left[G_{j}^{\|} b_{0}+G_{j}^{\perp} c_{0}+\mathcal{Q}_{j}^{*}\left(q_{\mathrm{gy}}\right)\right] \cdot \dot{r}-\left[q_{\|} G_{j}^{\|}+q_{\perp} G_{j}^{\perp}+\varrho_{j} \cdot \nabla \phi\right] \dot{t}+\mathcal{L}_{j}^{*}\left(q_{\mathrm{gy}}, \dot{q}_{\mathrm{gy}}\right),
\end{aligned}
$$

with

$$
\begin{aligned}
\mathcal{Q}_{j}^{*}\left(q_{\mathrm{gy}}\right) & :=\mathcal{R}_{j}\left(A_{0}\right)-\sum_{m=1}^{j}\left[G_{j-m}^{\|}\left(\varrho_{m} \times\left(\nabla \times b_{0}\right)\right)+G_{j-m}^{\perp}\left(\varrho_{m} \times\left(\nabla \times c_{0}\right)\right)\right] \\
& +\mathcal{R}_{j-1}\left(A_{1}\right)+\sum_{l=2}^{j}\left[G_{j-l}^{\|} \mathcal{R}_{l-1}\left(b_{0}\right)+G_{j-l}^{\perp} \mathcal{R}_{l-1}^{\alpha}\left(c_{0}\right)\right]-\sum_{m=1}^{j} G_{j-m}^{\perp} G_{m}^{\theta} a_{0},
\end{aligned}
$$


and

$$
\begin{aligned}
\mathcal{L}_{j}^{*}\left(q_{\mathrm{gy}}, \dot{q}_{\mathrm{gy}}\right): & =\sum_{l=1}^{j} \dot{\varrho}_{j+1-l} \cdot\left[\varrho_{l} \cdot \nabla A_{0}+\mathcal{R}_{l-1}\left(A_{0}\right)\right] \\
& -\sum_{m=1}^{j}\left[\dot{G}_{j-m}^{\|}\left(\varrho_{m} \cdot b_{0}\right)+\dot{G}_{j-m}^{\perp}\left(\varrho_{m} \cdot c_{0}\right)-G_{j-m}^{\perp} \dot{\alpha}\left(\varrho_{m} \cdot a_{0}\right)\right] \\
& +\sum_{l=1}^{j-1}\left[\sum_{m=1}^{j-l} G_{j-l-m}^{\|} \dot{\varrho}_{m}\right] \cdot\left[\varrho_{l} \cdot \nabla b_{0}+\mathcal{R}_{l-1}\left(b_{0}\right)\right] \\
& +\sum_{l=1}^{j-1}\left[\sum_{m=1}^{j-l} G_{j-l-m}^{\perp} \dot{\varrho}_{m}\right] \cdot\left[\varrho_{l} \cdot \nabla c_{0}-G_{l}^{\theta} a_{0}+\mathcal{R}_{l-1}^{\alpha}\left(c_{0}\right)\right] \\
& +\sum_{l=1}^{j-1} \dot{\varrho}_{j-l} \cdot\left[\varrho_{l} \cdot \nabla A_{1}+\mathcal{R}_{l-1}\left(A_{1}\right)\right]-\mathcal{R}_{j-1}(\phi) \dot{t} .
\end{aligned}
$$

Here, $\mathcal{R}$ and $\mathcal{R}^{\alpha}$ have been defined in (72) and (75), respectively, and we remind the reader of the sum convention (68).

\subsubsection{Gauge-invariant formulation}

It will be convenient to write (88) and (89) in terms of the electromagnetic fields $E, B$ rather than the potentials $\phi, A$. For this, consider the product rule on the tangent space,

$$
\frac{\mathrm{d}}{\mathrm{d} s}\left(\varrho_{j} \cdot A\right)=\dot{\varrho}_{j} \cdot A+\varrho_{j} \cdot \dot{A}=\dot{\varrho}_{j} \cdot A+\left(\dot{r} \cdot \nabla A+\dot{t} \partial_{t} A\right) \cdot \varrho_{j}
$$

where we used the tangent map to express $\dot{A}$. One can thus use the identity

$$
\nabla A \cdot \varrho_{j}-\varrho_{j} \cdot \nabla A=\varrho_{j} \times(\nabla \times A)=\varrho_{j} \times B
$$

to obtain

$$
\dot{\varrho}_{j} \cdot A+\varrho_{j} \cdot \nabla A \cdot \dot{r}=\frac{\mathrm{d}}{\mathrm{d} s}\left(\varrho_{j} \cdot A\right)-\dot{r} \cdot\left(\varrho_{j} \times B\right)-\dot{t} \partial_{t} A \cdot \varrho_{j}
$$

Therefore, by definition (3), one has the equivalence

$$
\begin{gathered}
\dot{\varrho}_{j} \cdot A_{0}+\varrho_{j} \cdot \nabla A_{0} \cdot \dot{r} \sim-\dot{r} \cdot\left(\varrho_{j} \times B_{0}\right) \\
\dot{\varrho}_{j} \cdot A_{1}+\varrho_{j} \cdot \nabla A_{1} \cdot \dot{r}-\varrho_{j} \cdot \nabla \phi \dot{t} \sim-\dot{r} \cdot\left(\varrho_{j} \times B_{1}\right)+\varrho_{j} \cdot E \dot{t} .
\end{gathered}
$$

The only remaining terms featuring the electromagnetic potentials are the first terms in each line of (90), as well as the first and the last line of (91). Since $\mathcal{R}$ is linear, these terms are of the generic form

$$
\mathcal{R}_{j-1}(A \cdot \dot{r}-\phi \dot{t})+\sum_{l=1}^{j-1} \dot{\varrho}_{j-l} \cdot\left[\varrho_{l} \cdot \nabla A+\mathcal{R}_{l-1}(A)\right]
$$


From (72) we write

$$
\mathcal{R}_{j-1}(A \cdot \dot{r}-\phi \dot{t})=\sum_{k_{1}=1}^{j-1} \frac{j-k_{1}}{j}\left(\varrho_{j-k_{1}} \cdot \nabla^{\prime}\right)\left(\varrho_{k_{1}} \cdot \nabla^{\prime}\right)(A \cdot \dot{r}-\phi \dot{t})+\mathcal{R}_{j-1}^{\mathrm{I}}(A \cdot \dot{r}-\phi \dot{t}),
$$

where for $j \geq 3$ we defined

$$
\begin{aligned}
& \mathcal{R}_{j-1}^{\mathrm{I}}(A \cdot \dot{r}-\phi \dot{t}):= \sum_{k_{1}=2}^{j-1} \sum_{k_{2}=1}^{k_{1}-1} \frac{\left(j-k_{1}\right)\left(k_{1}-k_{2}\right)}{j k_{1}} \\
& \times\left(\varrho_{j-k_{1}} \cdot \nabla^{\prime}\right)\left(\varrho_{k_{1}-k_{2}} \cdot \nabla^{\prime}\right)\left(\varrho_{k_{2}} \cdot \nabla^{\prime}\right)(A \cdot \dot{r}-\phi \dot{t})+\ldots \\
&+ \sum_{k_{1}=j-2 \geq 3}^{j-1} \sum_{k_{2}=j-3}^{k_{1}-1} \ldots \sum_{k_{j-2}=1}^{k_{j-3}-1} \frac{\left(j-k_{1}\right)\left(k_{1}-k_{2}\right) \ldots\left(k_{j-3}-k_{j-3}\right)}{j k_{1} k_{2} \ldots k_{j-3}} \\
&\left(\varrho_{j-k_{1}} \cdot \nabla^{\prime}\right)\left(\varrho_{k_{1}-k_{2}} \cdot \nabla^{\prime}\right) \ldots\left(\varrho_{k_{j-3}-k_{j-2}} \cdot \nabla^{\prime}\right)\left(\varrho_{k_{j-2}} \cdot \nabla^{\prime}\right)(A \cdot \dot{r}-\phi \dot{t}) \\
&+\frac{1}{j !}\left(\varrho_{1} \cdot \nabla^{\prime}\right)^{j}(A \cdot \dot{r}-\phi \dot{t}) .
\end{aligned}
$$

The first term in the sum of (94) can be written as

$$
\begin{aligned}
\sum_{l=1}^{j-1} \varrho_{l} \cdot \nabla A & \dot{\varrho}_{j-l}=\frac{1}{2} \sum_{l=1}^{j}\left(\varrho_{l} \cdot \nabla A \cdot \dot{\varrho}_{j-l}+\varrho_{j-l} \cdot \nabla A \cdot \varrho_{l}\right) \\
= & \frac{1}{2} \sum_{l=1}^{j-1}\left(\varrho_{l} \cdot \nabla A \cdot \dot{\varrho}_{j-l}-\dot{\varrho}_{j-l} \cdot \nabla A \cdot \varrho_{l}\right) \\
& +\frac{1}{2} \sum_{l=1}^{j-1}\left[\frac{\mathrm{d}}{\mathrm{d} s}\left(\varrho_{j-l} \cdot \nabla A \cdot \varrho_{l}\right)-\varrho_{j-l} \cdot \nabla\left(\dot{r} \cdot \nabla A+\dot{t} \partial_{t} A\right) \cdot \varrho_{l}\right] \\
= & \frac{1}{2} \sum_{l=1}^{j-1}\left[-\dot{\varrho}_{j-l} \cdot\left(\varrho_{l} \times B_{0}\right)+\frac{\mathrm{d}}{\mathrm{d} s}\left(\varrho_{j-l} \cdot \nabla A \cdot \varrho_{l}\right)-\varrho_{j-l} \cdot \nabla\left(\dot{r} \cdot \nabla A+\dot{t} \partial_{t} A\right) \cdot \varrho_{l}\right] .
\end{aligned}
$$

Moreover, in (95),

$$
\begin{aligned}
& \sum_{l=1}^{j-1} \frac{j-l}{j}\left(\varrho_{j-l} \cdot \nabla^{\prime}\right)\left(\varrho_{l} \cdot \nabla^{\prime}\right)(A \cdot \dot{r}-\phi \dot{t}) \\
= & \frac{1}{2} \sum_{l=1}^{j-1}\left[\frac{j-l}{j}\left(\varrho_{j-l} \cdot \nabla^{\prime}\right)\left(\varrho_{l} \cdot \nabla^{\prime}\right)+\frac{l}{j}\left(\varrho_{l} \cdot \nabla^{\prime}\right)\left(\varrho_{j-l} \cdot \nabla^{\prime}\right)\right](A \cdot \dot{r}-\phi \dot{t}) \\
= & \frac{1}{2} \sum_{l=1}^{j-1}\left(\varrho_{j-l} \cdot \nabla^{\prime}\right)\left(\varrho_{l} \cdot \nabla^{\prime}\right)(A \cdot \dot{r}-\phi \dot{t}) .
\end{aligned}
$$


Combining this result with the last term in the last line of (97) yields

$$
\begin{aligned}
& \frac{1}{2} \sum_{l=1}^{j-1}\left[\left(\varrho_{j-l} \cdot \nabla^{\prime}\right)\left(\varrho_{l} \cdot \nabla^{\prime}\right)(A \cdot \dot{r}-\phi \dot{t})-\varrho_{j-l} \cdot \nabla^{\prime}\left(\dot{r} \cdot \nabla A+\dot{t} \partial_{t} A\right) \cdot \varrho_{l}\right] \\
= & \frac{1}{2} \sum_{l=1}^{j-1}\left[\left(\varrho_{j-l} \cdot \nabla^{\prime}\right)\left(\varrho_{l} \cdot \nabla A-\nabla A \cdot \varrho_{l}\right) \cdot \dot{r}\right]-\frac{1}{2} \sum_{l=1}^{j-1} \varrho_{j-l} \cdot \nabla^{\prime}\left(\nabla \phi \dot{t}+\dot{t} \partial_{t} A\right) \cdot \varrho_{l} \\
= & \frac{1}{2} \sum_{l=1}^{j-1}\left[\left(\varrho_{j-l} \cdot \nabla B_{0}\right) \times \varrho_{l}\right] \cdot \dot{r}+\frac{1}{2} \sum_{l=1}^{j-1} \varrho_{j-l} \cdot \nabla E \cdot \varrho_{l} \dot{t} .
\end{aligned}
$$

Hence we arrived at

$$
\begin{aligned}
& \mathcal{R}_{j-1}(A \cdot \dot{r}-\phi \dot{t})+\sum_{l=1}^{j-1} \dot{\varrho}_{j-l} \cdot\left[\varrho_{l} \cdot \nabla A+\mathcal{R}_{l-1}(A)\right] \\
& \sim \frac{1}{2} \sum_{l=1}^{j-1}\left[\left(\varrho_{j-l} \cdot \nabla B\right) \times \varrho_{l}\right] \cdot \dot{r}+\frac{1}{2} \sum_{l=1}^{j-1} \varrho_{j-l} \cdot \nabla E \cdot \varrho_{l} \dot{t}-\frac{1}{2} \sum_{l=1}^{j-1} \dot{\varrho}_{j-l} \cdot\left(\varrho_{l} \times B\right) \\
& \quad+\mathcal{R}_{j-1}^{\mathrm{I}}(A \cdot \dot{r}-\phi \dot{t})+\sum_{l=1}^{j-1} \dot{\varrho}_{j-l} \cdot \mathcal{R}_{l-1}(A) .
\end{aligned}
$$

The terms in the last line still contain the electromagentic potentials instead of the fields. We were not able to prove that a field representation exists at all orders. However, we can easily prove it for $j=3$ :

$$
\begin{aligned}
\mathcal{R}_{2}^{\mathrm{I}}(A \cdot \dot{r}-\phi \dot{t})+\dot{\varrho}_{1} \cdot \mathcal{R}_{1}(A) & \\
= & \frac{1}{3 !}\left(\varrho_{1} \cdot \nabla^{\prime}\right)^{3}(A \cdot \dot{r}-\phi \dot{t})+\frac{1}{2 !}\left(\varrho_{1} \cdot \nabla^{\prime}\right)^{2} A \cdot \dot{\varrho}_{1} \\
= & \frac{1}{6}\left(\varrho_{1} \cdot \nabla^{\prime}\right)^{2}\left(\varrho_{1} \cdot \nabla(A \cdot \dot{r}-\phi \dot{t})+A \cdot \dot{\varrho}_{1}\right)+\frac{1}{3}\left(\varrho_{1} \cdot \nabla^{\prime}\right)^{2} A \cdot \dot{\varrho}_{1} \\
= & \frac{1}{6}\left(\varrho_{1} \cdot \nabla^{\prime}\right)^{2}\left[\varrho_{1} \cdot \nabla(A \cdot \dot{r}-\phi \dot{t})+\frac{\mathrm{d}}{\mathrm{d} s}\left(A \cdot \varrho_{1}\right)-\left(\dot{r} \cdot \nabla A+\dot{t} \partial_{t} A\right) \cdot \varrho_{1}\right]+\frac{1}{3}\left(\varrho_{1} \cdot \nabla^{\prime}\right)^{2} A \cdot \dot{\varrho}_{1} \\
= & -\frac{1}{6} \dot{r} \cdot\left[\varrho_{1} \times\left(\varrho_{1} \cdot \nabla^{\prime}\right)^{2} B\right]+\frac{1}{6}\left(\varrho_{1} \cdot \nabla^{\prime}\right)\left(\varrho_{1} \cdot \nabla E \cdot \varrho_{1}\right) \dot{t}+\frac{1}{6} \frac{\mathrm{d}}{\mathrm{d} s}\left[\left(\varrho_{1} \cdot \nabla^{\prime}\right)^{2} A \cdot \varrho_{1}\right] \\
& -\frac{1}{3}\left(\varrho_{1} \cdot \nabla^{\prime}\right)\left(\dot{\varrho}_{1} \cdot \nabla A \cdot \varrho_{1}\right)+\frac{1}{3}\left(\varrho_{1} \cdot \nabla^{\prime}\right)^{2} A \cdot \dot{\varrho}_{1} \\
\sim & -\frac{1}{6} \dot{r} \cdot\left[\varrho_{1} \times\left(\varrho_{1} \cdot \nabla^{\prime}\right)^{2} B\right]+\frac{1}{6}\left(\varrho_{1} \cdot \nabla^{\prime}\right)\left(\varrho_{1} \cdot \nabla E \cdot \varrho_{1}\right) \dot{t}-\frac{1}{3} \dot{\varrho}_{1} \cdot\left[\varrho_{1} \times\left(\varrho_{1} \cdot \nabla B\right)\right] .
\end{aligned}
$$

We conjecture that such field representations can be derived at every order and leave the proof for later. 
In summary, the above algebra leads to the following representation of (88) and (89), respectively,

$$
\varphi_{0}^{(1)}(0) \sim-\dot{r} \cdot\left(\varrho_{1} \times B_{0}\right),
$$

and, for $n \geq 1$ (switching the index $j$ to $n$ ),

$$
\begin{aligned}
\frac{\varphi_{0}^{(n+1)}(0)}{(n+1) !}+ & \frac{\varphi_{1}^{(n)}(0)}{(n) !}-\frac{\varphi_{2}^{(n)}(0)}{(n) !} \dot{t} \sim\left[G_{n}^{\|} b_{0}+G_{n}^{\perp} c_{0}-\varrho_{n+1} \times B_{0}-\varrho_{n} \times B_{1}+\mathcal{Q}_{n}\left(q_{\mathrm{gy}}\right)\right] \cdot \dot{r} \\
& -\left(\varrho_{n} \cdot b_{0}\right) \dot{q}_{\|}-G_{n-1}^{\theta} q_{\perp}\left(\dot{\varrho}_{1} \cdot a_{0}\right)-\left[q_{\|} G_{n}^{\|}+q_{\perp} G_{n}^{\perp}-\varrho_{n} \cdot E\right] \dot{t}+\mathcal{L}_{n}\left(q_{\mathrm{gy}}, \dot{q}_{\mathrm{gy}}\right)
\end{aligned}
$$

with

$$
\begin{aligned}
\mathcal{Q}_{n}\left(q_{\mathrm{gy}}\right):= & \frac{1}{2} \sum_{l=1}^{n}\left(\varrho_{n+1-l} \cdot \nabla B_{0}\right) \times \varrho_{l}+\frac{1}{2} \sum_{l=1}^{n-1}\left(\varrho_{n-l} \cdot \nabla B_{1}\right) \times \varrho_{l} \\
& \quad-\sum_{m=1}^{n}\left[G_{n-m}^{\|}\left(\varrho_{m} \times\left(\nabla \times b_{0}\right)\right)+G_{n-m}^{\perp}\left(\varrho_{m} \times\left(\nabla \times c_{0}\right)\right)\right] \\
& +\sum_{l=2}^{n}\left[G_{n-l}^{\|} \mathcal{R}_{l-1}\left(b_{0}\right)+G_{n-l}^{\perp} \mathcal{R}_{l-1}^{\alpha}\left(c_{0}\right)\right]-\sum_{m=1}^{n} G_{n-m}^{\perp} G_{m}^{\theta} a_{0} \\
\mathcal{L}_{n}\left(q_{\mathrm{gy}}, \dot{q}_{\mathrm{gy}}\right):= & -\frac{1}{2} \sum_{l=1}^{n} \dot{\varrho}_{n+1-l} \cdot\left(\varrho_{l} \times B_{0}\right)-\frac{1}{2} \sum_{l=1}^{n-1} \dot{\varrho}_{n-l} \cdot\left(\varrho_{l} \times B_{1}\right)+\frac{1}{2} \sum_{l=1}^{n-1} \varrho_{n-l} \cdot \nabla E \cdot \varrho_{l} \dot{t} \\
- & \sum_{m=1}^{n}\left[\dot{G}_{n-m}^{\|}\left(\varrho_{m} \cdot b_{0}\right)+\dot{G}_{n-m}^{\perp}\left(\varrho_{m} \cdot c_{0}\right)-G_{n-m}^{\perp} \dot{\alpha}\left(\varrho_{m} \cdot a_{0}\right)\right] \\
+ & \sum_{l=1}^{n-1}\left[\sum_{m=1}^{n-l} G_{n-l-m}^{\|} \dot{\varrho}_{m}\right] \cdot\left[\varrho_{l} \cdot \nabla b_{0}+\mathcal{R}_{l-1}\left(b_{0}\right)\right] \\
+ & \sum_{l=1}^{n-1}\left[\sum_{m=1}^{n-l} G_{n-l-m}^{\perp} \dot{\varrho}_{m}\right] \cdot\left[\varrho_{l} \cdot \nabla c_{0}-G_{l}^{\theta} a_{0}+\mathcal{R}_{l-1}^{\alpha}\left(c_{0}\right)\right] \\
+ & \mathcal{R}_{n}^{\mathrm{I}}\left(A_{0}\right) \cdot \dot{r}+\sum_{l=2}^{n} \dot{\varrho}_{n+1-l} \cdot \mathcal{R}_{l-1}\left(A_{0}\right)+\mathcal{R}_{n-1}^{\mathrm{I}}\left(A_{1} \cdot \dot{r}-\phi \dot{t}\right)+\sum_{l=2}^{n-1} \dot{\varrho}_{n-l} \cdot \mathcal{R}_{l-1}\left(A_{1}\right) .
\end{aligned}
$$

Here, the expressions for $\mathcal{R}, \mathcal{R}^{\alpha}$ and $\mathcal{R}^{\mathrm{I}}$ are given in (72), (75) and (96), respectively.

\subsection{Proof of Theorem 1}

The Lagrangian (44) can be written as

$$
L_{\mathrm{gy}}^{(N)}=\frac{1}{\varepsilon} L_{-1}^{*}+L_{0}^{*}+\sum_{n=1}^{N} \varepsilon^{n} L_{n}^{*},
$$


with

$$
L_{-1}^{*}=A_{0} \cdot \dot{r}, \quad L_{0}^{*}=\left(q_{\|} b_{0}+A_{1}\right) \cdot \dot{r}-\left(\frac{q_{\|}^{2}}{2}+\frac{q_{\perp}^{2}}{2}+\phi\right) \dot{t}, \quad L_{n}^{*}=\Gamma_{n} \dot{\alpha},
$$

and $\Gamma_{1}=\mu=q_{\perp}^{2} /\left(2\left|B_{0}\right|\right)$. On the other hand, the series expansion of the Lagrangian $L^{\varepsilon}$ in Proposition 1 is composed of the terms

$$
L_{-1}=A_{0} \cdot \dot{r}, \quad L_{0}=\left(q_{\|} b_{0}+q_{\perp} c_{0}-\varrho_{1} \times B_{0}+A_{1}\right) \cdot \dot{r}-\left(\frac{q_{\|}^{2}}{2}+\frac{q_{\perp}^{2}}{2}+\phi\right) \dot{t},
$$

and

$$
\begin{aligned}
L_{1 \leq n \leq N}= & {\left[G_{n}^{\|} b_{0}+G_{n}^{\perp} c_{0}-\varrho_{n+1} \times B_{0}-\varrho_{n} \times B_{1}+\mathcal{Q}_{n}\right] \cdot \dot{r} } \\
& -\left(q_{\|} G_{n}^{\|}+q_{\perp} G_{n}^{\perp}-\varrho_{n} \cdot E\right) \dot{t}+\mathcal{L}_{n},
\end{aligned}
$$

where $\mathcal{Q}_{n}$ and $\mathcal{L}_{n}$ are given in (100) and (101), respectively. We shall show that generators can be chosen such that $L_{n} \sim L_{n}^{*}$ for $-1 \leq n \leq N$. At lowest order one has $L_{-1}=L_{-1}^{*}$ and nothing needs to be done. At zeroth order we choose

$$
\varrho_{1}=\frac{q_{\perp}}{\left|B_{0}\right|} b_{0} \times c_{0}=\frac{q_{\perp}}{\left|B_{0}\right|} a_{0},
$$

which yields $L_{0}=L_{0}^{*}$. For the higher orders we proof the following:

Lemma 9. For $n \geq 1$ one can choose generators $\mathbf{G}_{n}, \varrho_{n+1, \perp}$ in the Lagrangian (103), where $\varrho_{n+1}=\left(b_{0} \cdot \varrho_{n+1}\right) b_{0}+\varrho_{n+1, \perp}$, such that $L_{n} \sim L_{n}^{*}=\Gamma_{n} \dot{\alpha}$ for arbitrary functions $b_{0} \cdot \varrho_{n+1}$ and $G_{n}^{\theta}$. Moreover, $\Gamma_{1}=\mu=q_{\perp}^{2} /\left(2\left|B_{0}\right|\right)$ and $\widetilde{\Gamma}_{n}=0$ for all $n$.

Proof. We proceed by induction. For $n=1$ we have

$$
\begin{aligned}
& \mathcal{Q}_{1}=\frac{1}{2}\left(\varrho_{1} \cdot \nabla B_{0}\right) \times \varrho_{1}-q_{\|} \varrho_{1} \times\left(\nabla \times b_{0}\right)-q_{\perp} \varrho_{1} \times\left(\nabla \times c_{0}\right)-q_{\perp} G_{1}^{\theta} a_{0}, \\
& \mathcal{L}_{1}=-\frac{1}{2} \dot{\varrho}_{1} \cdot\left(\varrho_{1} \times B_{0}\right)-\dot{q}_{\|}\left(\varrho_{1} \cdot b_{0}\right)-\dot{q}_{\perp}\left(\varrho_{1} \cdot c_{0}\right)+q_{\perp} \dot{\alpha}\left(\varrho_{1} \cdot a_{0}\right) .
\end{aligned}
$$

Now, from the result (104) for $\varrho_{1}$ we compute

$$
\dot{\varrho}_{1}=\frac{\dot{q}_{\perp}}{\left|B_{0}\right|} a_{0}-\frac{q_{\perp}}{\left|B_{0}\right|^{2}}\left(\dot{r} \cdot \nabla\left|B_{0}\right|\right) a_{0}+\frac{q_{\perp}}{\left|B_{0}\right|} \dot{r} \cdot \nabla a_{0}+\frac{q_{\perp}}{\left|B_{0}\right|} \dot{\alpha} c_{0} .
$$

Moreover, from $a_{0} \times b_{0}=c_{0}$,

$$
\dot{\varrho}_{1} \cdot\left(\varrho_{1} \times B_{0}\right)=q_{\perp} \dot{\varrho}_{1} \cdot c_{0}=\frac{q_{\perp}^{2}}{\left|B_{0}\right|} \dot{r} \cdot \nabla a_{0} \cdot c_{0}+\frac{q_{\perp}^{2}}{\left|B_{0}\right|} \dot{\alpha},
$$

where we recognize the gyro-gauge $\mathrm{R}=\nabla a_{0} \cdot c_{0}=\nabla e_{2} \cdot e_{1}$. Inserting this into $\mathcal{L}_{1}$ gives

$$
\begin{aligned}
L_{1}= & \left(G_{1}^{\|} b_{0}+G_{1}^{\perp} c_{0}-\varrho_{2} \times B_{0}-\frac{q_{\perp}}{\left|B_{0}\right|} a_{0} \times B_{1}+\mathcal{Q}_{1}-\frac{q_{\perp}^{2}}{2\left|B_{0}\right|} \mathrm{R}\right) \cdot \dot{r} \\
& -\left(q_{\|} G_{1}^{\|}+q_{\perp} G_{1}^{\perp}-\frac{q_{\perp}}{\left|B_{0}\right|} a_{0} \cdot E\right) \dot{t}+\frac{q_{\perp}^{2}}{2\left|B_{0}\right|} \dot{\alpha} .
\end{aligned}
$$


We can eliminate the Hamiltonian multiplying $\dot{t}$ by setting

$$
G_{1}^{\perp}=-\frac{q_{\|}}{q_{\perp}} G_{1}^{\|}+\frac{1}{\left|B_{0}\right|} a_{0} \cdot E
$$

and write the remainder of $L_{1}$ as

$$
L_{1}=\left(G_{1}^{\|} b_{0}-\varrho_{2} \times B_{0}+\gamma_{1}\right) \cdot \dot{r}+\frac{q_{\perp}^{2}}{2\left|B_{0}\right|} \dot{\alpha} .
$$

Any vector $v \in \mathbb{R}^{3}$ can be written as $v=v_{\|} b_{0}+v_{\perp}$, where $v_{\|}=v \cdot b_{0}$ and $v_{\perp}=b_{0} \times v \times b_{0}$; hence by setting

$$
\varrho_{2, \perp}=\frac{b_{0} \times \gamma_{1}}{\left|B_{0}\right|}, \quad G_{1}^{\|}=-\gamma_{1, \|}
$$

we obtain

$$
L_{1}=\frac{q_{\perp}^{2}}{2\left|B_{0}\right|} \dot{\alpha}=\Gamma_{1} \dot{\alpha}=L_{1}^{*} .
$$

Moreover, $b_{0} \cdot \varrho_{2}$ and $G_{1}^{\theta}$ are still arbitrary; thus we proved that the statement of the lemma holds for $n=1$.

Suppose now that the statement holds for some $n \geq 1$. From Proposition 1 we write the Lagrangian at order $n+1$ as

$$
\begin{aligned}
L_{n+1}= & {\left[G_{n+1}^{\|} b_{0}+G_{n+1}^{\perp} c_{0}-\varrho_{n+2} \times B_{0}-\varrho_{n+1} \times B_{1}+\mathcal{Q}_{n+1}\right] \cdot \dot{r} } \\
& -\left(q_{\|} G_{n+1}^{\|}+q_{\perp} G_{n+1}^{\perp}-\varrho_{n+1} \cdot E\right) \dot{t}+\mathcal{L}_{n+1},
\end{aligned}
$$

Let us examine the term $\mathcal{L}_{n+1}$ from (101) a bit more careful; in particular, let us single out two terms:

- the term $-\dot{q}_{\|}\left(\varrho_{n+1} \cdot b_{0}\right)$ from the second line $(m=n+1)$,

- the term $-q_{\perp} G_{n}^{\theta}\left(\dot{\varrho}_{1} \cdot a_{0}\right)$ from the fourth line $(l=n)$.

From (107) we obtain

$$
\dot{\varrho}_{1} \cdot a_{0}=\frac{\dot{q}_{\perp}}{\left|B_{0}\right|}-\frac{q_{\perp}}{\left|B_{0}\right|^{2}}\left(\dot{r} \cdot \nabla\left|B_{0}\right|\right) .
$$

Therefore,

$$
\mathcal{L}_{n+1}=-\dot{q}_{\|}\left(\varrho_{n+1} \cdot b_{0}\right)-\frac{q_{\perp}}{\left|B_{0}\right|} G_{n}^{\theta} \dot{q}_{\perp}+\text { terms } .
$$

Moreover, let us add to the Lagrangian $L_{n+1}$ the "total time derivative" of some arbitrary function $S_{n+1}: \Omega_{\mathrm{gy}} \rightarrow \mathbb{R}$, and let us write it in compact notation similarly to (111),

$$
\begin{aligned}
L_{n+1} & \sim\left[G_{n+1}^{\|} b_{0}-\varrho_{n+2} \times B_{0}+\gamma_{n+1, r}\right] \cdot \dot{r}-\left(q_{\perp} G_{n+1}^{\perp}+\gamma_{n+1, t}\right) \dot{t} \\
& -\left(\varrho_{n+1} \cdot b_{0}+\gamma_{n+1, \|}\right) \dot{q}_{\|}-\left(\frac{q_{\perp}}{\left|B_{0}\right|} G_{n}^{\theta}+\gamma_{n+1, \perp}\right) \dot{q}_{\perp}+\left(\frac{\partial S_{n+1}}{\partial \alpha}+\gamma_{n+1, \alpha}\right) \dot{\alpha},
\end{aligned}
$$

where all remaining terms have been gathered in the linear form $\gamma_{n+1}$. Let us treat each component of the Poincaré-Cartan form on the right-hand-side of (115) separately: 
- The component of $\dot{r}$ is zero for

$$
\varrho_{n+2, \perp}=\frac{b_{0} \times \gamma_{n+1}}{\left|B_{0}\right|}, \quad G_{n+1}^{\|}=-\gamma_{1, \|} .
$$

- The component of $\dot{t}$ (i.e. the Hamiltonian) is zero for

$$
G_{n+1}^{\perp}=-\frac{\gamma_{n+1, t}}{q_{\perp}}
$$

- Since $b_{0} \cdot \varrho_{n+1}$ is still undetermined by the inductive hypothesis, the component of $\dot{q}_{\|}$ is zero for

$$
b_{0} \cdot \varrho_{n+1}=-\gamma_{n+1, \|} \cdot
$$

- Noting that $G_{n}^{\theta}$ is still undetermined by the inductive hypothesis, the component of $\dot{q}_{\perp}$ is zero for

$$
G_{n}^{\theta}=-\frac{\left|B_{0}\right|}{q_{\perp}} \gamma_{n+1, \perp} .
$$

- The term with $\dot{\alpha}$ is rewritten as

$$
\left(\frac{\partial S_{n+1}}{\partial \alpha}+\gamma_{n+1, \alpha}\right) \dot{\alpha}=\left(\frac{\partial S_{n+1}}{\partial \alpha}+\left\langle\gamma_{n+1, \alpha}\right\rangle+\widetilde{\gamma_{n+1, \alpha}}\right) \dot{\alpha},
$$

where $\gamma_{n+1, \alpha}$ has been decomposed into gyro-average and fluctuations. The equation

$$
\frac{\partial S_{n+1}}{\partial \alpha}+\widetilde{\gamma_{n+1, \alpha}}=0
$$

has $2 \pi$-periodic solutions $S_{n+1}$. We pick one of those solutions to obtain

$$
\left(\frac{\partial S_{n+1}}{\partial \alpha}+\gamma_{n+1, \alpha}\right) \dot{\alpha}=\left\langle\gamma_{n+1, \alpha}\right\rangle \dot{\alpha} .
$$

Hence, with the above choices for the generators, all that remains from (115) is

$$
L_{n+1} \sim\left\langle\gamma_{n+1, \alpha}\right\rangle \dot{\alpha}=: \Gamma_{n+1} \dot{\alpha}=L_{n+1}^{*} .
$$

Noting that $b_{0} \cdot \varrho_{n+2}$ and $G_{n+1}^{\theta}$ are still arbitrary and that $\widetilde{\Gamma}_{n+1}=0$, we showed that the statement of the lemma holds for $n+1$ and thus completed the proof by induction.

Considering the regularity of the generators $\mathbf{G}_{n}$ the following is true:

Lemma 10. For $1 \leq n \leq N$ we have $\mathbf{G}_{n} \in C^{N+2-n}\left(\Omega_{\mathrm{gy}}\right)$ and $\varrho_{n+1, \perp} \in C^{N+2-n}\left(\Omega_{\mathrm{gy}}\right)$. 
Proof. The proof is again achieved by induction. For $n=1$ the generators $\varrho_{1}, G_{1}^{\|}, G_{1}^{\perp}$ and $\varrho_{2, \perp}$ are given in Lemma 7 and $G_{1}^{\theta}$ is given in Lemma 8, respectively. From assumption 1 we deduce $\mathbf{G}_{1}, \varrho_{2, \perp} \in C^{N+1}\left(\Omega_{\mathrm{gy}}\right)$. Assuming the statement holds for some $n \leq N-1$, it follows from the proof of Lemma 9 that the generators $\varrho_{n+2, \perp}, G_{n+1}^{\|}, G_{n+1}^{\perp}$ and $b_{0} \cdot \varrho_{n+1}$ have the same regularity as the Lagrangian $L_{n+1}$ written in (114). The fact that $L_{n+1} \in C^{N+2-(n+1)}$ follows from $\dot{\varrho}_{n+1, \perp} \in C^{N+2-n-1}$ due to the inductive hypothesis (needed in the first term of $\mathcal{L}_{n+1}$, equation (101)) as well as from $\mathcal{R}_{n}\left(b_{0}\right), \mathcal{R}_{n}^{\alpha}\left(c_{0}\right), \mathcal{R}_{n+1}^{\mathrm{I}}\left(A_{0}\right)$ all being in $C^{N+2-(n+1)}$.

It remains to determine the regularity of the gererator $G_{n+1}^{\theta}$, which is the same as the one of the terms multplying $\dot{q}_{\perp}$ in $\mathcal{L}_{n+2}$, according to the proof of Lemma 9. A close inspection of (101) reveals that such terms can only stem from $\dot{\varrho}_{n+2, \perp}, \dot{\varrho}_{n+1}, \dot{G}_{n+1}^{\|}$and $\dot{G}_{n+1}^{\perp}$. But derivation with respect to $q_{\perp}$ does not change the regularity since everything is $C^{\infty}$ in the velocities; therefore, $G_{n+1}^{\theta} \in C^{N+2-(n+1)}$ and the proof is complete.

Taking the statement from Lemma 10 for $n=N$ we have $\mathbf{G}_{N} \in C^{2}\left(\Omega_{\text {gy }}\right)$ and on the next level $\varrho_{N+1, \perp} \in C^{2}\left(\Omega_{\mathrm{gy}}\right)$. According to Lemma 9 all other generators at the level $N+1$ can be set to zero and thus Theorem 1 is proved.

\subsection{Proof of Lemma 4}

Lemma 11. (Gronwall [40]) Suppose that for $t \in\left[t_{0}, t_{0}+T\right]$

$$
\varphi(t) \leq b\left(t-t_{0}\right)+a \int_{t_{0}}^{t} \varphi(s) d s+c,
$$

with $\varphi(t)$ continuous, $\varphi(t) \geq 0$ for $t \in\left[t_{0}, t_{0}+T\right]$ and constants $a>0, b, c \geq 0$, then

$$
\varphi(t) \leq\left(\frac{b}{a}+c\right) e^{a\left(t-t_{0}\right)}-\frac{b}{a}
$$

for $t \in\left[t_{0}, t_{0}+T\right]$.

In order to set the framework necessary to apply Gronwall's lemma, let us write (54) as integral equations,

$$
\begin{aligned}
& \mathbf{z}(t)=\mathbf{z}_{0}+\int_{t_{0}}^{t}\left[\Lambda(\mathbf{z}, s, \varepsilon)+\varepsilon^{N} S(\mathbf{z}, s, \varepsilon)\right] d s \\
& \overline{\mathbf{z}}(t)=\overline{\mathbf{z}}_{0}+\int_{t_{0}}^{t} \Lambda(\overline{\mathbf{z}}, s, \varepsilon) d s
\end{aligned}
$$

Subtracting the equations and taking the norm yields

$$
\begin{aligned}
\| \overline{\mathbf{z}}(t) & -\mathbf{z}(t)\|=\| \overline{\mathbf{z}}_{0}-\mathbf{z}_{0}+\int_{t_{0}}^{t}\left[\Lambda(\overline{\mathbf{z}}, s, \varepsilon)-\Lambda(\mathbf{z}, s, \varepsilon)-\varepsilon^{N} S(\mathbf{z}, \alpha, s, \varepsilon)\right] d s \| \\
& \leq\left\|\overline{\mathbf{z}}_{0}-\mathbf{z}_{0}\right\|+\int_{t_{0}}^{t}\|\Lambda(\overline{\mathbf{z}}, s, \varepsilon)-\Lambda(\mathbf{z}, s, \varepsilon)\| d s+\varepsilon^{N} \int_{t_{0}}^{t}\|S(\mathbf{z}, \alpha, s, \varepsilon)\| d s .
\end{aligned}
$$


The residual $S$ is continuous; a solution $\mathbf{z}(s)$ of $\left(P^{\varepsilon}\right)$ is too (remark 2) and lives in a bounded domain with size $O(1)$ as $\varepsilon \rightarrow 0$. Therefore,

$$
\|S(\mathbf{z}(s), \alpha, s, \varepsilon)\| \leq\|S\|_{\infty, \varepsilon}:=\max _{\hat{\Omega}_{\mathrm{gy}} \times\left(0, \varepsilon_{\max }\right)}\|S(\mathbf{z}, \alpha, s, \varepsilon)\|=O(1) .
$$

Moreover, $\Lambda$ is Lipschitz with constant $\ell_{\Lambda}$ and we may estimate

$$
\|\overline{\mathbf{z}}(t)-\mathbf{z}(t)\| \leq\left\|\overline{\mathbf{z}}_{0}-\mathbf{z}_{0}\right\|+\ell_{\Lambda} \int_{t_{0}}^{t}\|\overline{\mathbf{z}}(s)-\mathbf{z}(s)\| d s+\varepsilon^{N}\left(t-t_{0}\right)\|S\|_{\infty} .
$$

We now apply Gronwall's lemma with $\varphi(t)=\|\overline{\mathbf{z}}(t)-\mathbf{z}(t)\|, a=\ell_{\Lambda}, b=\varepsilon^{N}\|S\|_{\infty, \varepsilon}$ and $c=\left\|\overline{\mathbf{z}}_{0}-\mathbf{z}_{0}\right\|$ to obtain

$$
\|\overline{\mathbf{z}}(t)-\mathbf{z}(t)\| \leq\left(\varepsilon^{N} \frac{\|S\|_{\infty, \varepsilon}}{\ell_{\Lambda}}+\left\|\overline{\mathbf{z}}_{0}-\mathbf{z}_{0}\right\|\right) e^{\ell_{\Lambda}\left(t-t_{0}\right)}-\varepsilon^{N} \frac{\|S\|_{\infty, \varepsilon}}{\ell_{\Lambda}} .
$$

\section{Conclusion}

Gyrokinetics is a prevalent theory in plasma physics; it enables the numerical simulation of sophisticated multiscale physics on long timescales. The contribution of this work is to build a mathematically sound foundation for gyrokinetics by means of averaging systems of differential equations on the level of the Lagrangian function, hence the name variational averaging (VA). The formal theory of VA is well-known for almost three decades; the most important results with emphasis on applications in plasma physics have been gathered in recent reviews $[9,30]$. The theory has here been made rigorous in the following sense:

- The theory starts from the normalized set of equations (9) and stays consistent with this scaling thorough all orders of the perturbation expansion.

- The gyro-transformations (37) employed in this work exist, c.f. Theorem 1. This is in contrast to the formal theories, where transformations are infinite series of which the convergence cannot be established.

- We state the unambiguous definition of a gyrokinetic equation in (51) by means of the "decoupled dynamics" (50), which stem from a truncated Lagrangian function.

- For the first time we give an error estimate for gyrokinetics in Theorem 2.

The method of VA is thus well-established for the charged particle motion. It seems plausible that this technique could be applied also to other problems of averaging, where the Hamiltonian structure of the equations is important and which are non-canonical symplectic, i.e. with a Lagrangian of the form (13). For instance, an application of VA to the semi-classical limit of the Schrödinger equation could be envisioned. But also other fields 
like liquid crystal dynamics might be an interesting possibility for application of the VAmethod. Moreover, the relation between VA and other averaging methods, in particular normal forms, should be clarified.

Regarding the charged particle, let us comment on some of the practical implications of the here derived results. In view of the GY-Lagrangian from Theorem 1, repeated in equation (48) with the generalized magnetic moment $\widehat{\mu}$ as one of the coordinates, we remark that only the Hamiltonian $H_{\text {gy }}$ depends on the order $N$ of the perturbation expansion, whereas the symplectic form remains unchanged through all orders. This is remarkable because we did not make any particular effort to achieve this; in conventional GY-theories this is usually enforced by an ansatz for the GY-transformation in the form of a Lie-series. Here, the formalism is considerably simpler.

Expressions for the Hamiltonian $H_{\text {gy }}$ have been computed for $N=2$ in the Lemmas 6 and 8. They differ from the conventional GY-Hamiltonians as was pointed out in Remark 5. This is not a surprise considering the amount of freedom within the VA methodology: at each order $n$, there is a choice to be made which terms of the Lagrangian $L_{n}$ in the series (43) should be attached to the generators, and thus appear in the transformation, and which should be kept in the Lagrangian, and thus appear in the dynamics. In conventional GYtheories the gyro-average of the Lagrangian constitutes the dynamics, while the fluctuating part disappears into the generators. However, this is not mandatory. Our approach was to attach as many terms as possible to the generators, even gyro-averaged terms, thereby keeping the dynamics simpler. This could be beneficial for a certain class of numerical codes, in particular particle-based codes, in which an efficient particle pusher is important. We plan the implementation of such a scheme in a forthcoming work.

Finally, the error estimate in Theorem 2 relies on the the assumption that the gyrokinetic initial condition has gyro-fluctuations of the order $O\left(\varepsilon^{N}\right)$; this is called a "wellprepared" initial condition. In the estimate we compare the solution of the averaged part (52) of the gyrokinetic equation to the solution of the Vlasov equation, transformed to the new coordinates, which depends on the gyro-angle $\alpha$. It is thus clear that the error is small only when the $\alpha$-dependence of the Vlasov solution $f$ is. In practice one is often faced with the computation of velocity moments of $f$, which is why we chose to focus on the estimate from Theorem 2.

\section{Acknowledgements}

I am thankful to Eric Sonnendrücker for encouraging this work and for his valuable scientific input. I also thank Francis Filbet, Michael Kraus and Edoardo Zoni for the inspiring discussion I had with them as well as Roman Hatzky for reading the manuscript with care.

\section{References}

[1] R. Abraham and J.E. Marsden. Foundations of Mechanics. Addison-Wesley, 1978. 
[2] V.I. Arnold. Mathematical Methods of Classical Mechanics. Number 60 in Graduate Texts in Mathematics. Springer, 2nd edition, 1989.

[3] M. Bostan. The Vlasov-Maxwell system with strong initial magnetic field: guidingcenter approximation. Multiscale Modeling \& Simulation, 6(3):1026-1058, 2007.

[4] M. Bostan. Gyrokinetic Vlasov equation in three dimensional setting. Second order approximation. Multiscale Modeling \& Simulation, 8(5):1923-1957, 2010.

[5] M. Bostan. Transport equations with disparate advection fields. Application to the gyrokinetic models in plasma physics. Journal of Differential Equations, 249(7):1620$1663,2010$.

[6] M. Bostan. MultiScale Analysis for Linear First Order PDEs. The Finite Larmor Radius Regime . SIAM Journal on Mathematical Analysis, 48(3):2133-2188, 2016.

[7] A. Bottino, B. Scott, S. Brunner, B. F. McMillan, T. M. Tran, T. Vernay, L. Villard, S. Jolliet, R. Hatzky, and A. G. Peeters. Global nonlinear electromagnetic simulations of tokamak turbulence. IEEE Transactions on Plasma Science, 38(9):2129-2135, 2010.

[8] A. Brizard. Nonlinear gyrokinetic Maxwell-Vlasov equations using magnetic coordinates. Journal of plasma physics, 41(3):541-559, 1989.

[9] A.J. Brizard and T.S. Hahm. Foundations of nonlinear gyrokinetic theory. Rev. Mod. Phys., 79:421, 2007.

[10] J.W. Burby, J. Squire, and H. Qin. Automation of the guiding center expansion. Physics of Plasmas, 20:072105, 2013.

[11] J. Candy and R.E. Waltz. Anomalous transport scaling in the DIII-D tokamak matched by supercomputer simulation. Physical review letters, 91(4):045001, 2003.

[12] J. R. Cary and A. J. Brizard. Hamiltonian theory of guiding-center motion. Reviews of modern physics, 81(2):693, 2009.

[13] P. Chartier, N. Crouseilles, and M. Lemou. An averaging technique for transport equations. arXiv preprint arXiv:1609.09819, 2016.

[14] P. Chartier, A. Murua, and J. M. Sanz-Serna. A formal series approach to averaging: exponentially small error estimates. Discrete and Continuous Dynamical SystemsSeries A, 32(9), 2012.

[15] P. Degond and F. Filbet. On the Asymptotic Limit of the Three Dimensional VlasovPoisson System for Large Magnetic Field: Formal Derivation. Journal of Statistical Physics, 165(4):765-784, 2016.

[16] T. Frankel. The geometry of physics: an introduction. Cambridge University Press, 2011. 
[17] E. Frénod and E. Sonnendrücker. Homogenization of the Vlasov equation and of the Vlasov-Poisson system with a strong external magnetic field. Asymptotic Analysis, 18(3-4):193-213, 1998.

[18] E. Frénod and E. Sonnendrücker. The finite Larmor radius approximation. SIAM Journal on Mathematical Analysis, 32(6):1227-1247, 2001.

[19] F. Golse and L. Saint-Raymond. The Vlasov-Poisson system with strong magnetic field. Journal de mathématiques pures et appliquées, 78(8):791-817, 1999.

[20] T. Görler, X. Lapillonne, S. Brunner, T. Dannert, F. Jenko, F. Merz, and D. Told. The global version of the gyrokinetic turbulence code GENE. Journal of Computational Physics, 230(18):7053-7071, 2011.

[21] V. Grandgirard, Y. Sarazin, X. Garbet, G. Dif-Pradalier, Ph. Ghendrih, N. Crouseilles, G. Latu, E. Sonnendrücker, N. Besse, and P. Bertrand. GYSELA, a full-f global gyrokinetic Semi-Lagrangian code for ITG turbulence simulations. AIP Conference Proceedings, 871(1):100-111, 2006.

[22] T.S. Hahm. Nonlinear gyrokinetic equations for tokamak microturbulence. The Physics of fluids, 31(9):2670-2673, 1988.

[23] T.S. Hahm. Nonlinear gyrokinetic equations for turbulence in core transport barriers. Physics of Plasmas, 3(12):4658-4664, 1996.

[24] E. Hairer, C. Lubich, and G. Wanner. Geometric Numerical Integration, volume 31. Springer Series in Computational Mathematics, 2006.

[25] D. Han-Kwan. The three-dimensional finite Larmor radius approximation. Asymptotic Analysis, 66(1):9-33, 2010.

[26] R. D. Hazeltine and J. D. Meiss. Plasma Confinement. Dover Books, 2003.

[27] J.A. Heikkinen, S. Henriksson, S. Janhunen, T.P. Kiviniemi, and F. Ogando. Gyrokinetic simulation of particle and heat transport in the presence of wide orbits and strong profile variations in the edge plasma. Contributions to Plasma Physics, 46(79):490-495, 2006.

[28] J. D. Jackson. Classical electrodynamics. John Wiley \& Sons, 2007.

[29] G. Jost, T.M. Tran, W.A. Cooper, L. Villard, and K. Appert. Global linear gyrokinetic simulations in quasi-symmetric configurations. Physics of Plasmas, 8(7):3321-3333, 2001.

[30] J. A. Krommes. The gyrokinetic description of microturbulence in magnetized plasmas. Annual Review of Fluid Mechanics, 44:175-201, 2012. 
[31] M. Kruskal. Asymptotic Theory of Hamiltonian and other Systems with all Solutions Nearly Periodic. J. Math. Phys., 3:806, 1962.

[32] Z. Lin, T. S. Hahm, W.W. Lee, W. M. Tang, and R. B. White. Turbulent transport reduction by zonal flows: Massively parallel simulations. Science, 281(5384):18351837, 1998.

[33] R.G. Littlejohn. A guiding center Hamiltonian: A new approach. J. Math. Phys., 20(12), 1979.

[34] R.G. Littlejohn. Variational principles of guiding center motion. J. Plasma Physics, 29:111-125, 1983.

[35] R. G. Littlejohn. Hamiltonian formulation of guiding center motion. The Physics of Fluids, 24(9):1730-1749, 1981.

[36] R. G. Littlejohn. Hamiltonian perturbation theory in noncanonical coordinates. Journal of Mathematical Physics, 23(5):742-747, 1982.

[37] T. G. Northrop. Adiabatic charged-particle motion. Reviews of Geophysics, 1(3):283304, 1963.

[38] S.E. Parker, Y. Chen, W. Wan, B.I. Cohen, and W.M. Nevins. Electromagnetic gyrokinetic simulations. Physics of Plasmas, 11(5):2594-2599, 2004.

[39] F. I. Parra and I. Calvo. Phase-space Lagrangian derivation of electrostatic gyrokinetics in general geometry. Plasma Physics and Controlled Fusion, 53(4):045001, 2011.

[40] J. A. Sanders, F. Verhulst, and J. A. Murdock. Averaging methods in nonlinear dynamical systems, volume 59. Springer, 2007.

[41] B. D. Scott. Gyrokinetic Field Theory as a Gauge Transform or: gyrokinetic theory without Lie transforms. arXiv preprint arXiv:1708.06265, 2017.

[42] C. Tronci. From liquid crystal models to the guiding-center theory of magnetized plasmas. Annals of Physics, 371:323-337, 2016.

[43] N. Tronko and A.J. Brizard. Lagrangian and Hamiltonian constraints for guidingcenter Hamiltonian theories. Physics of Plasmas, 22(11):112507, 2015.

[44] N. Tronko, A. Bottino, C. Chandre, and E. Sonnendruecker. Hierarchy of second order gyrokinetic Hamiltonian models for particle-in-cell codes. Plasma Physics and Controlled Fusion, 59(6):064008, 2017. 